\title{
Enantioselective $\alpha$-Arylation of Ketones with Aryl Triflates Catalyzed by Difluorphos Complexes of Palladium and Nickel
}

\author{
Xuebin liao, Zhiqiang Weng and John F. Hartwig* \\ Department of Chemistry, University of Illinois at Urbana-Champaign, 600 S. Mathews Avenue, \\ Urbana, IL 61801 and Department of Chemistry, Yale University, P.O. Box 208107, New Haven, \\ Connecticut 06520-8107 \\ E-mail: jhartwig@scs.uiuc.edu
}

Supporting Information

\section{Table of Contents}

General Experimental procedures, and product isolation and characterization

Preparation of palladium and nickel complexes

S17-20

Studies of stoichiometric reactions of arylmetal halide complexes with enolates

S20-23

Determination of absolute configuration

S23-25 
General Method. All catalytic reactions were conducted in sealed vials, and the reactions were assembled in a nitrogen-filled glovebox. The synthesis of arylpalladium halide and arylnickel halide complexes was conducted in a nitrogen-filled glovebox. $\left\{\mathrm{Pd}\left[\mathrm{P}(o-\mathrm{Tol})_{3}\right](\mathrm{Br})(p-\right.$ Tol) $\}_{2}$ (95.4 mg, $\left.0.0820 \mathrm{mmol}\right)$ was prepared according to a literature procedure. ${ }^{1}$ Aryl triflates were prepared as described previously. ${ }^{2}$ All other chemicals were used as received from commercial sources. Pd(dba) $)_{2}$, segphos, BINAP, P-phos and difluorphos were purchased from Strem Chemical Co. and used as received. Toluene were distilled under argon from sodium / benzophenone prior to use. ${ }^{1} \mathrm{H}$ NMR spectra were obtained on a 400 - or $500-\mathrm{MHz}$ spectrometer, and chemical shifts were recorded relative to residual protiated solvent. ${ }^{13} \mathrm{C}$ NMR spectra were obtained at 100.6 or $125.8 \mathrm{MHz}$ on a $400-$ or $500-\mathrm{MHz}$ instrument, and chemical shifts were recorded relative to the solvent resonance. Both ${ }^{1} \mathrm{H}$ NMR and ${ }^{13} \mathrm{C}$ NMR chemical shifts are reported in parts per million downfield from tetramethylsilane. Chromatographic purifications were performed by flash chromatography using silica gel (200-400 mesh) or preparative TLC. The yields of the coupled products included in all tables refer to isolated yields. Enantioselectivities were measured by HPLC using the indicated columns and conditions.

\section{Representative Procedure for the Asymmetric Arylation of Ketones with Aryl Triflates}

using Pd(0)/Difluorphos (Method A). To a screw-capped vial containing $(R)$-difluorphos $(8.2$ $\mathrm{mg}, 0.012 \mathrm{mmol}), \mathrm{Pd}(\mathrm{dba})_{2}(5.8 \mathrm{mg}, 0.010 \mathrm{mmol}), \mathrm{NaO}^{\prime} \mathrm{Bu}(19.2 \mathrm{mg}, 0.200 \mathrm{mmol})$ and the ketone $(0.100 \mathrm{mmol})$ in toluene $(2.0 \mathrm{~mL})$ was added the aryl triflate $(0.200 \mathrm{mmol})$. The vial was sealed with a cap containing a PTFE septum and removed from the drybox. The reaction mixture was stirred at $60^{\circ} \mathrm{C}$ for $48-72 \mathrm{~h}$. The crude reaction was then cooled to room temperature and quenched with ice water. The resulting solution was then diluted with ethyl acetate $(15 \mathrm{~mL})$ and washed with brine. The organic phase with dried over $\mathrm{Na}_{2} \mathrm{SO}_{4}$, filtered, and concentrated at 
reduced pressure. Pure product was obtained from the residue by preparative TLC with the eluent described.

Representative Procedure for the Asymmetric Arylation of Ketones with Aryl Triflates Using Ni(0)/Difluorphos (Method B). To a screw-capped vial containing $(R)$-difluorphos (10.3 $\mathrm{mg}, 0.0150 \mathrm{mmol}), \mathrm{Ni}(\mathrm{COD})_{2}(3.5 \mathrm{mg}, 0.016 \mathrm{mmol}), \mathrm{NaO}^{t} \mathrm{Bu}(48.0 \mathrm{mg}, 0.500 \mathrm{mmol})$ and the ketone $(0.250 \mathrm{mmol})$ in toluene $(2.0 \mathrm{~mL})$ was added the aryl triflate $(0.750 \mathrm{mmol})$. The vial was sealed with a cap containing a PTFE septum and removed from drybox. The reaction mixture was stirred at $80{ }^{\circ} \mathrm{C}$ for $60 \mathrm{~h}$. The crude reaction was then cooled to room temperature and quenched with ice water. The resulting solution was then diluted with ethyl acetate $(20 \mathrm{~mL})$ and washed with brine. The organic phase was dried over $\mathrm{Na}_{2} \mathrm{SO}_{4}$, filtered, and concentrated at reduced pressure. The residue was then purified by chromatography on silica gel, eluting with hexane/ether to provide the product as indicated.

Representative Procedure for the Asymmetric Arylation of Ketones with Aryl Triflates Using Ni(0)/Difluorphos (Method C). To a screw-capped vial containing $(R)$-difluorphos (10.3 $\mathrm{mg}, 0.0150 \mathrm{mmol}), \mathrm{Ni}(\mathrm{COD})_{2}(3.5 \mathrm{mg}, 0.016 \mathrm{mmol}), \mathrm{NaO}^{t} \mathrm{Bu}(72.0 \mathrm{mg}, 0.750 \mathrm{mmol})$ and ketone $(0.250 \mathrm{mmol})$ in toluene $(1.0 \mathrm{~mL})$ was added the aryl triflate $(0.100 \mathrm{mmol})$. The vial was sealed with a cap containing a PTFE septum and removed from drybox. The reaction mixture was stirred at $100{ }^{\circ} \mathrm{C}$ for $80 \mathrm{~h}$. The crude reaction was then cooled to room temperature and quenched with ice water. The resulting solution was then diluted with ethyl acetate $(20 \mathrm{~mL})$ and washed with brine. The organic phase was dried over $\mathrm{Na}_{2} \mathrm{SO}_{4}$, filtered, and concentrated at reduced pressure. The residue was then purified by chromatography on silica gel, eluting with hexane/ether to provide the product as indicated yield. 
2-Methyl-2-(4-t-butylphenyl)-1-tetralone (Table 2, Entry 2). Method A was followed. The reaction mixture was stirred for $48 \mathrm{~h}$ and was purified by preparative TLC (hexane/ether $=$ 97:3) to provide the title compound as white solid in $85 \%$ yield. The ee was determined to be 92\% by chiral HPLC analysis (Chiralcel OJ-H column; solvent, hexane/isopropanol (99/1); flow rate, $0.50 \mathrm{~mL} / \mathrm{min} ; \mathrm{UV}$ lamp, $254 \mathrm{~nm}) .[\alpha]^{26}{ }_{\mathrm{D}}=164\left(\mathrm{c}=0.40, \mathrm{CHCl}_{3}\right) .{ }^{1} \mathrm{H} \mathrm{NMR}\left(\mathrm{CDCl}_{3}, 500\right.$ MHz): $\delta 1.26(9 \mathrm{H}, \mathrm{s}), 1.52(3 \mathrm{H}, \mathrm{s}), 2.22-2.28(1 \mathrm{H}, \mathrm{m}), 2.60(1 \mathrm{H}, \mathrm{dt}, \mathrm{J}=13.9,4.1 \mathrm{~Hz}), 2.77-2.89$ $(2 \mathrm{H}, \mathrm{m}), 7.11-7.15(2 \mathrm{H}, \mathrm{m}), 7.26-7.32(4 \mathrm{H}, \mathrm{m}), 7.41(1 \mathrm{H}, \mathrm{td}, \mathrm{J}=7.6,1.4 \mathrm{~Hz}), 8.16(1 \mathrm{H}, \mathrm{dd}, \mathrm{J}=$ 7.9, $1.5 \mathrm{~Hz}){ }^{13} \mathrm{C} \mathrm{NMR}\left(\mathrm{CDCl}_{3}, 125 \mathrm{MHz}\right): \delta 26.4,27.3,31.5,34.5,36.5,50.3,125.7,126.2$, $126.8,128.2,128.9,132.9,133.3,139.1,144.0,149.6,201.7$.

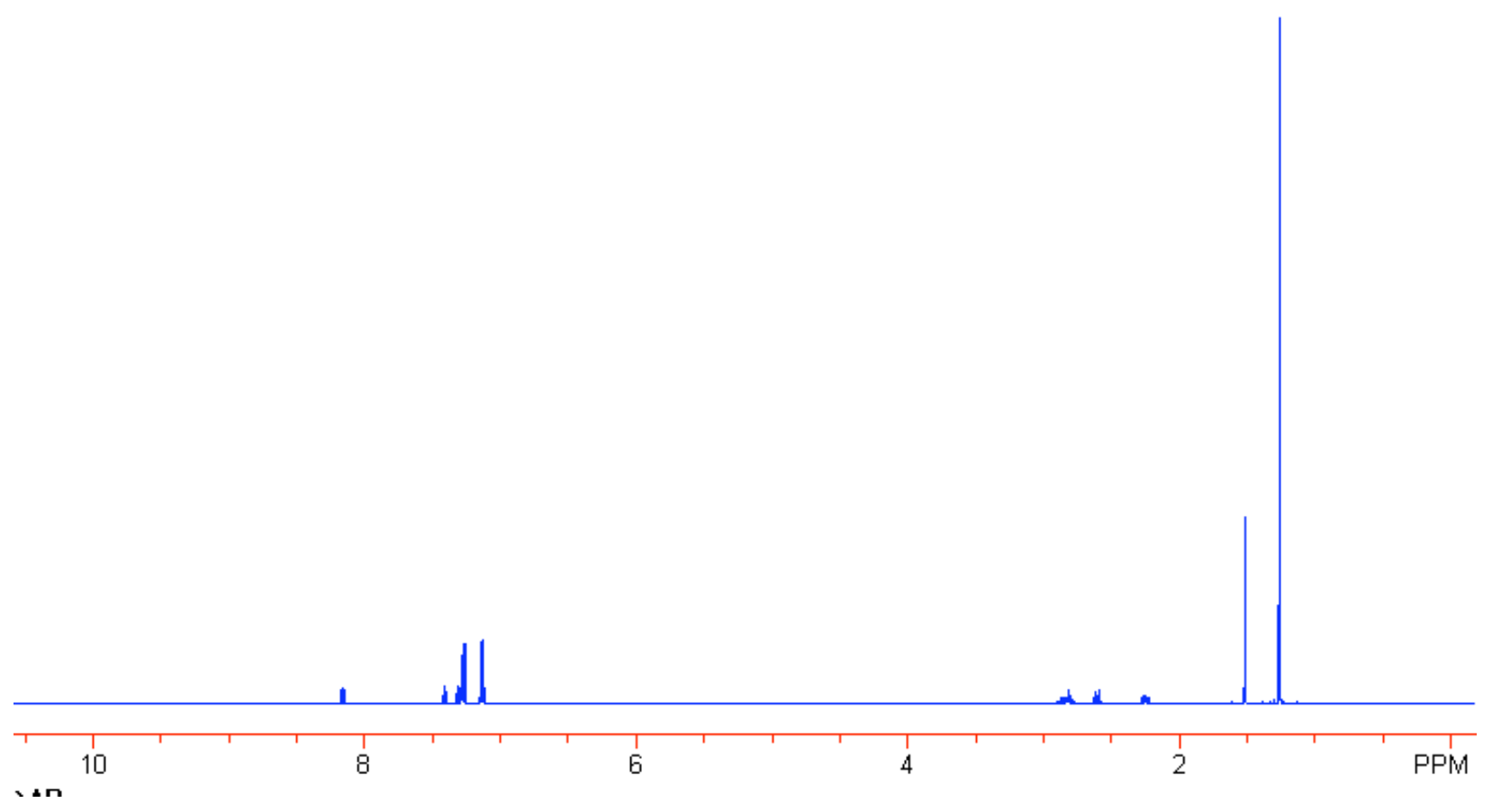




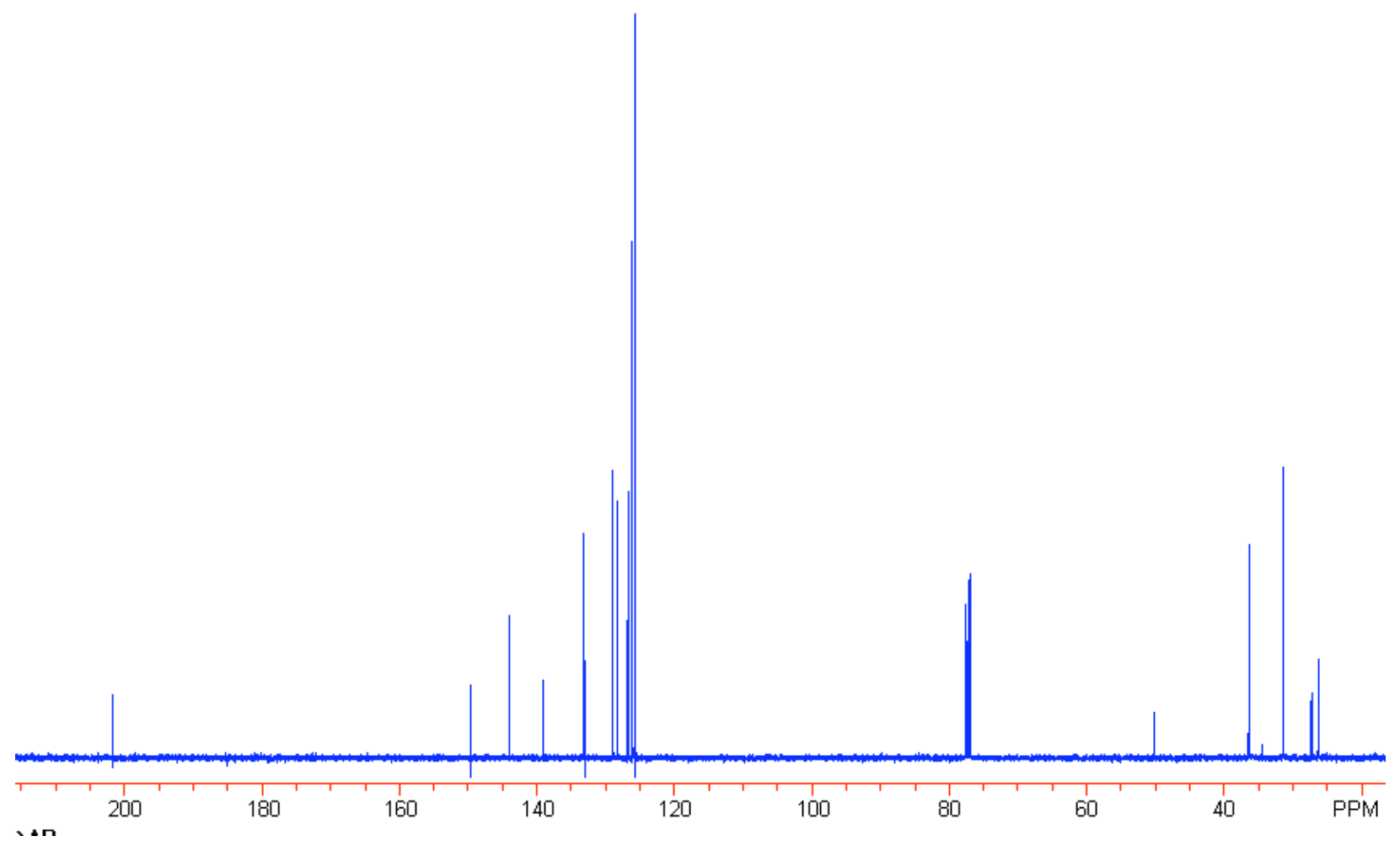

2-Methyl-2-(4-methylphenyl)-1-tetralone (Table 2, Entry 3). Method A was followed. The reaction mixture was stirred for $48 \mathrm{~h}$ and was purified by preparative TLC (hexane/ether $=$ 97:3) to provide the title compound in $79 \%$ yield. . The ee was determined to be $92 \%$ by chiral HPLC analysis (Chiralcel AD-H column; solvent, hexane/isopropanol (98/2); flow rate, 0.50 $\mathrm{mL} / \mathrm{min}$; UV lamp, $254 \mathrm{~nm}) .[\alpha]^{26}{ }_{\mathrm{D}}=233.7\left(\mathrm{c}=0.19, \mathrm{CHCl}_{3}\right) .{ }^{1} \mathrm{H}$ NMR $\left(\mathrm{CDCl}_{3}, 400 \mathrm{MHz}\right)$ : ठ $1.54(3 \mathrm{H}, \mathrm{s}), 2.21-2.30(1 \mathrm{H}, \mathrm{m}), 2.30(3 \mathrm{H}, \mathrm{s}), 2.62(1 \mathrm{H}, \mathrm{dt}, \mathrm{J}=14.0,3.9 \mathrm{~Hz}), 2.82-2.89(2 \mathrm{H}$, m), 7.09-7.16 (5H, m), $7.32(1 \mathrm{H}, \mathrm{t}, \mathrm{J}=7.5 \mathrm{~Hz}), 7.42(1 \mathrm{H}, \mathrm{td}, \mathrm{J}=7.4,1.5 \mathrm{~Hz}), 8.19(1 \mathrm{H}, \mathrm{dd}, \mathrm{J}=$ 7.8, $1.5 \mathrm{~Hz}) .{ }^{13} \mathrm{C} \mathrm{NMR}\left(\mathrm{CDCl}_{3}, 100 \mathrm{MHz}\right): \delta 21.2,26.4,27.5,36.3,50.4,126.5,126.8,128.2$, $128.9,129.5,132.9,133.3,136.5,139.2,143.8,201.7$. 

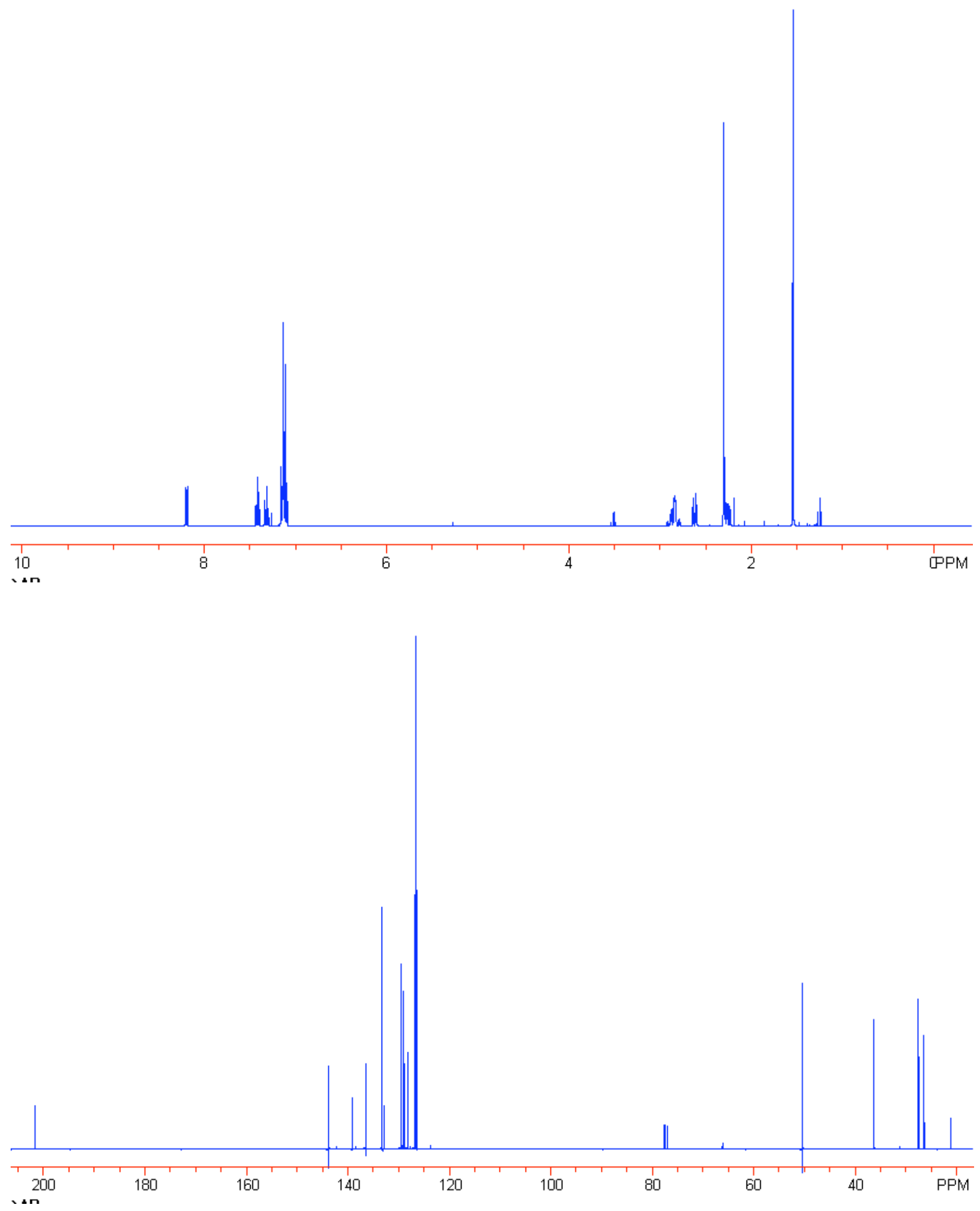

2-Methyl-2-(3,5-di-t-butylphenyl)-1-tetralone (Table 2, Entry 4). Method A was followed. The reaction mixture was stirred for $48 \mathrm{~h}$ and was purified by preparative $\mathrm{TLC}$ (hexane/ether $=$ 
97:3) to provide the title compound as a white solid in $83 \%$ yield. . The ee was determined to be 95\% by chiral HPLC analysis (Chiralcel AD-H column; solvent, hexane/isopropanol (99/1); flow rate, $0.50 \mathrm{~mL} / \mathrm{min}$; UV lamp, $254 \mathrm{~nm}) .[\alpha]^{26}=236\left(\mathrm{c}=0.11, \mathrm{CHCl}_{3}\right) .{ }^{1} \mathrm{H}$ NMR $\left(\mathrm{CDCl}_{3}, 500\right.$ MHz): $\delta 1.24(18 \mathrm{H}, \mathrm{s}), 1.53(3 \mathrm{H}, \mathrm{s}), 2.22-2.29(1 \mathrm{H}, \mathrm{m}), 2.63(1 \mathrm{H}, \mathrm{dt}, \mathrm{J}=14.1,3.9 \mathrm{~Hz}), 2.70-2.90$ $(2 \mathrm{H}, \mathrm{m}), 7.05(2 \mathrm{H}, \mathrm{d}, \mathrm{J}=1.7 \mathrm{~Hz}), 7.11(1 \mathrm{H}, \mathrm{d}, \mathrm{J}=6.0 \mathrm{~Hz}), 7.23(1 \mathrm{H}, \mathrm{t}, \mathrm{J}=1.7 \mathrm{~Hz}), 7.29(1 \mathrm{H}, \mathrm{t}, \mathrm{J}$ $=7.6 \mathrm{~Hz}), 7.39(1 \mathrm{H}, \mathrm{td}, \mathrm{J}=7.3,1.4 \mathrm{~Hz}), 8.13(1 \mathrm{H}, \mathrm{dd}, \mathrm{J}=7.8,1.0 \mathrm{~Hz}) \cdot{ }^{13} \mathrm{C} \mathrm{NMR}\left(\mathrm{CDCl}_{3}, 125\right.$ MHz): $\delta 26.3,27.5,31.6,35.1,36.3,50.8,120.7,120.8,126.7,128.0,128.8,133.0,133.3,140.9$, 143.7, 150.7, 202.0. Anal. Calcd. For $\mathrm{C}_{25} \mathrm{H}_{32} \mathrm{O}: \mathrm{C}, 86.15 ; \mathrm{H}, 9.25$. Found: C, 85.99; H, 9.51.

2-Methyl-2-(3,4-di-methoxylphenyl)-1-tetralone (Table 2, Entry 5). Method A was followed. The reaction mixture was stirred for $72 \mathrm{~h}$ and was purified by preparative TLC (hexane/ether $=80: 20)$ to provide the title compound in $79 \%$ yield. . The ee was determined to be $91 \%$ by chiral HPLC analysis (Chiralcel AD-H column; solvent, hexane/isopropanol (90/10); flow rate, $0.5 \mathrm{~mL} / \mathrm{min}$; UV lamp, $254 \mathrm{~nm}) .[\alpha]^{26}{ }_{\mathrm{D}}=193\left(\mathrm{c}=0.24, \mathrm{CHCl}_{3}\right) .{ }^{1} \mathrm{H} \mathrm{NMR}\left(\mathrm{CDCl}_{3}, 400\right.$ MHz): $\delta 1.50(3 \mathrm{H}, \mathrm{s}), 2.18-2.27(1 \mathrm{H}, \mathrm{m}), 2.56(1 \mathrm{H}, \mathrm{dt}, \mathrm{J}=14.2,3.9 \mathrm{~Hz}), 2.75-2.92(2 \mathrm{H}, \mathrm{m}), 3.79$ $(6 \mathrm{H}, \mathrm{s}), 6.72-6.74(3 \mathrm{H}, \mathrm{m}), 7.09(1 \mathrm{H}, \mathrm{d}, \mathrm{J}=7.6 \mathrm{~Hz}), 7.27(1 \mathrm{H}, \mathrm{t}, \mathrm{J}=7.7 \mathrm{~Hz}), 7.39(1 \mathrm{H}, \mathrm{t}, \mathrm{J}=7.0$ $\mathrm{Hz}), 8.12(1 \mathrm{H}, \mathrm{d}, \mathrm{J}=7.8 \mathrm{~Hz}){ }^{13} \mathrm{C} \mathrm{NMR}\left(\mathrm{CDCl}_{3}, 100 \mathrm{MHz}\right): \delta$ 26.3, 27.4, 36.4, 50.3, 56.0, 56.1, 110.0, 111.2, 118.9, 126.8, 128.1, 128.9, 132.8, 133.3, 134.5, 143.8, 147.9, 149.1, 201.6. Anal. Calcd. For $\mathrm{C}_{19} \mathrm{H}_{20} \mathrm{O}_{3}$ : C, 77.00; H, 6.80. Found: C, 76.91; H, 6.80.

2-Methyl-2-phenyl-1-indanone (Table 2, Entry 6). Method A was followed. The reaction mixture was stirred for $40 \mathrm{~h}$ and was purified by preparative TLC (hexane/ether $=97: 3$ ) to provide the title compound in $77 \%$ yield. . The ee was determined to be $70 \%$ by chiral HPLC analysis (Chiralcel AD-H column; solvent, hexane/isopropanol (99/1); flow rate, $0.50 \mathrm{~mL} / \mathrm{min}$; UV lamp, $254 \mathrm{~nm}) .[\alpha]^{26}=-34.9\left(\mathrm{c}=0.48, \mathrm{CHCl}_{3}\right) .{ }^{1} \mathrm{H} \mathrm{NMR}\left(\mathrm{CDCl}_{3}, 400 \mathrm{MHz}\right): \delta 1.68(3 \mathrm{H}, \mathrm{s})$, 
$3.32(1 \mathrm{H}, \mathrm{d}, \mathrm{J}=17.6 \mathrm{~Hz}), 3.61(1 \mathrm{H}, \mathrm{d}, \mathrm{J}=17.3 \mathrm{~Hz}), 7.21-7.27(1 \mathrm{H}, \mathrm{m}), 7.28-7.36(4 \mathrm{H}, \mathrm{m}), 7.43$ $(1 \mathrm{H}, \mathrm{t}, \mathrm{J}=7.5 \mathrm{~Hz}), 7.51(1 \mathrm{H}, \mathrm{d}, \mathrm{J}=7.7 \mathrm{~Hz}), 7.65(1 \mathrm{H}, \mathrm{t}, \mathrm{J}=7.4 \mathrm{~Hz}), 7.84(1 \mathrm{H}, \mathrm{d}, \mathrm{J}=7.6 \mathrm{~Hz}) .{ }^{13} \mathrm{C}$ NMR $\left(\mathrm{CDCl}_{3}, 100 \mathrm{MHz}\right): \delta \quad 24.6,45.1,53.3,125.1,126.4,126.6,126.9,128.0,128.8,135.4$, 135.8, 144.1, 152.9, 209.0.

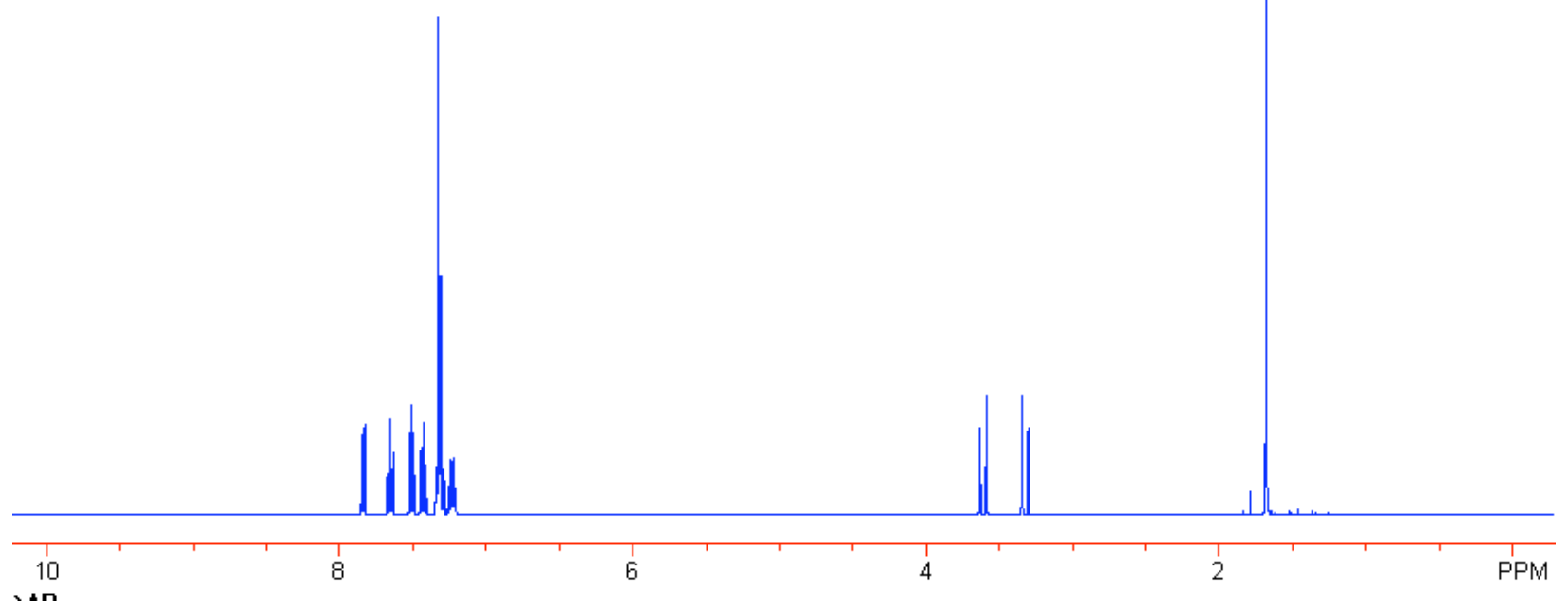




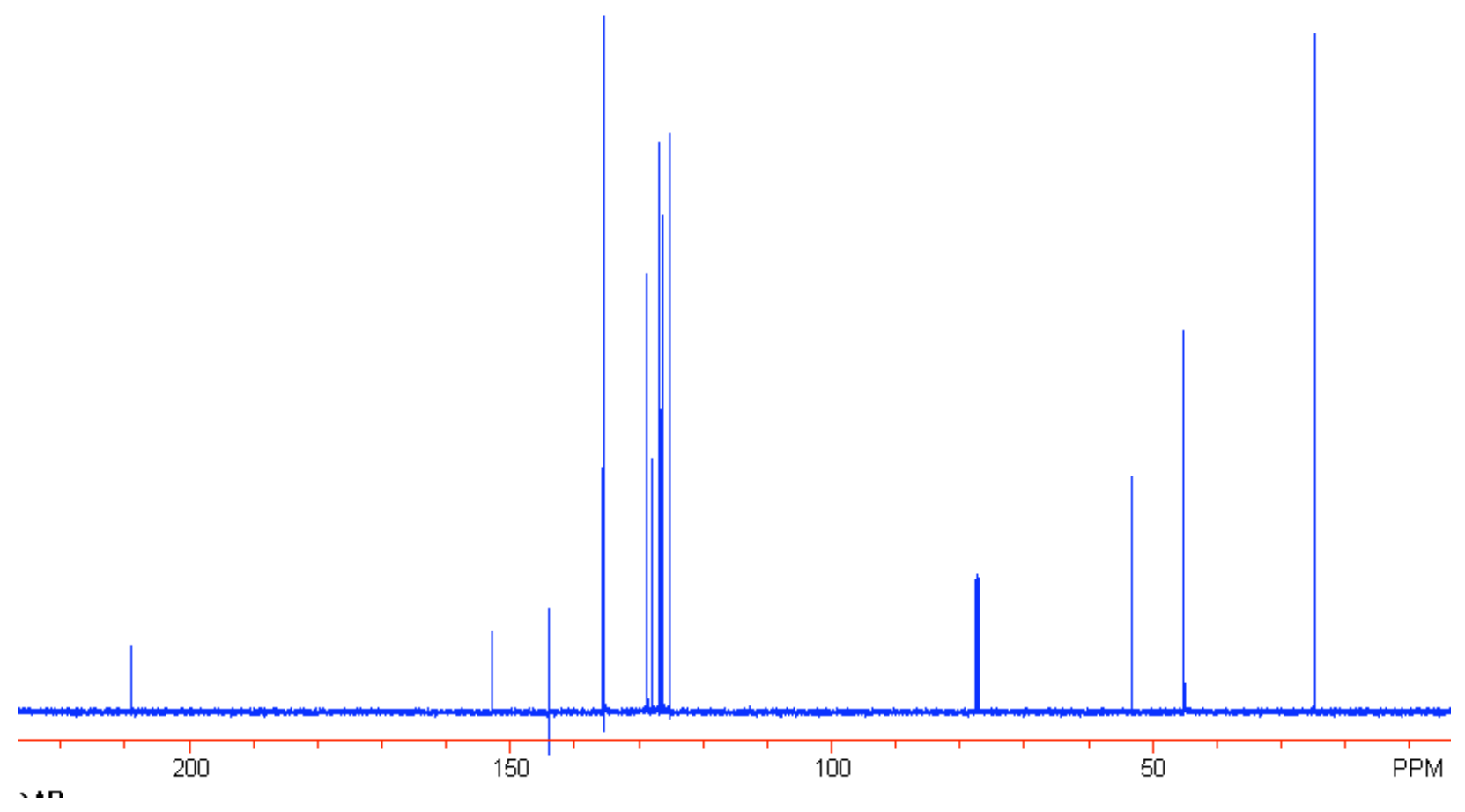

2-Methyl-2-(4-methylphenyl)-1-indanone (Table 2, Entry 7). Method A was followed and the reaction mixture was stirred for $40 \mathrm{~h}$, then purified by preparative TLC (hexane/ether $=97: 3$ ) to provide the title compound in $79 \%$ yield. . The ee was determined to be $78 \%$ by chiral HPLC analysis (Chiralcel AD-H column; solvent, hexane/isopropanol (98.5/1.5); flow rate, 1.0 $\mathrm{mL} / \mathrm{min}$; UV lamp, $254 \mathrm{~nm}) .[\alpha]^{26}{ }_{\mathrm{D}}=-35.2\left(\mathrm{c}=0.25, \mathrm{CHCl}_{3}\right) .{ }^{1} \mathrm{H} \mathrm{NMR}\left(\mathrm{CDCl}_{3}, 400 \mathrm{MHz}\right)$ : ठ $1.65(3 \mathrm{H}, \mathrm{s}), 2.30(3 \mathrm{H}, \mathrm{s}), 3.30(1 \mathrm{H}, \mathrm{d}, \mathrm{J}=17.3 \mathrm{~Hz}), 3.59(1 \mathrm{H}, \mathrm{d}, \mathrm{J}=17.3 \mathrm{~Hz}), 7.11(2 \mathrm{H}, \mathrm{d}, \mathrm{J}=$ $8.5 \mathrm{~Hz}), 7.20(2 \mathrm{H}, \mathrm{d}, \mathrm{J}=8.3 \mathrm{~Hz}), 7.42(1 \mathrm{H}, \mathrm{t}, \mathrm{J}=7.4 \mathrm{~Hz}), 7.49(1 \mathrm{H}, \mathrm{d}, \mathrm{J}=7.8 \mathrm{~Hz}), 7.64(1 \mathrm{H}, \mathrm{t}, \mathrm{J}$ $=7.5 \mathrm{~Hz}), 7.81(1 \mathrm{H}, \mathrm{d}, \mathrm{J}=7.5 \mathrm{~Hz}) .{ }^{13} \mathrm{C} \mathrm{NMR}\left(\mathrm{CDCl}_{3}, 100 \mathrm{MHz}\right): \delta$ 21.2, 24.6, 45.1, 53.1, 125.1, $126.2,126.6,127.9,129.5,135.3,135.9,136.5,141.0,152.8,209.1$. 

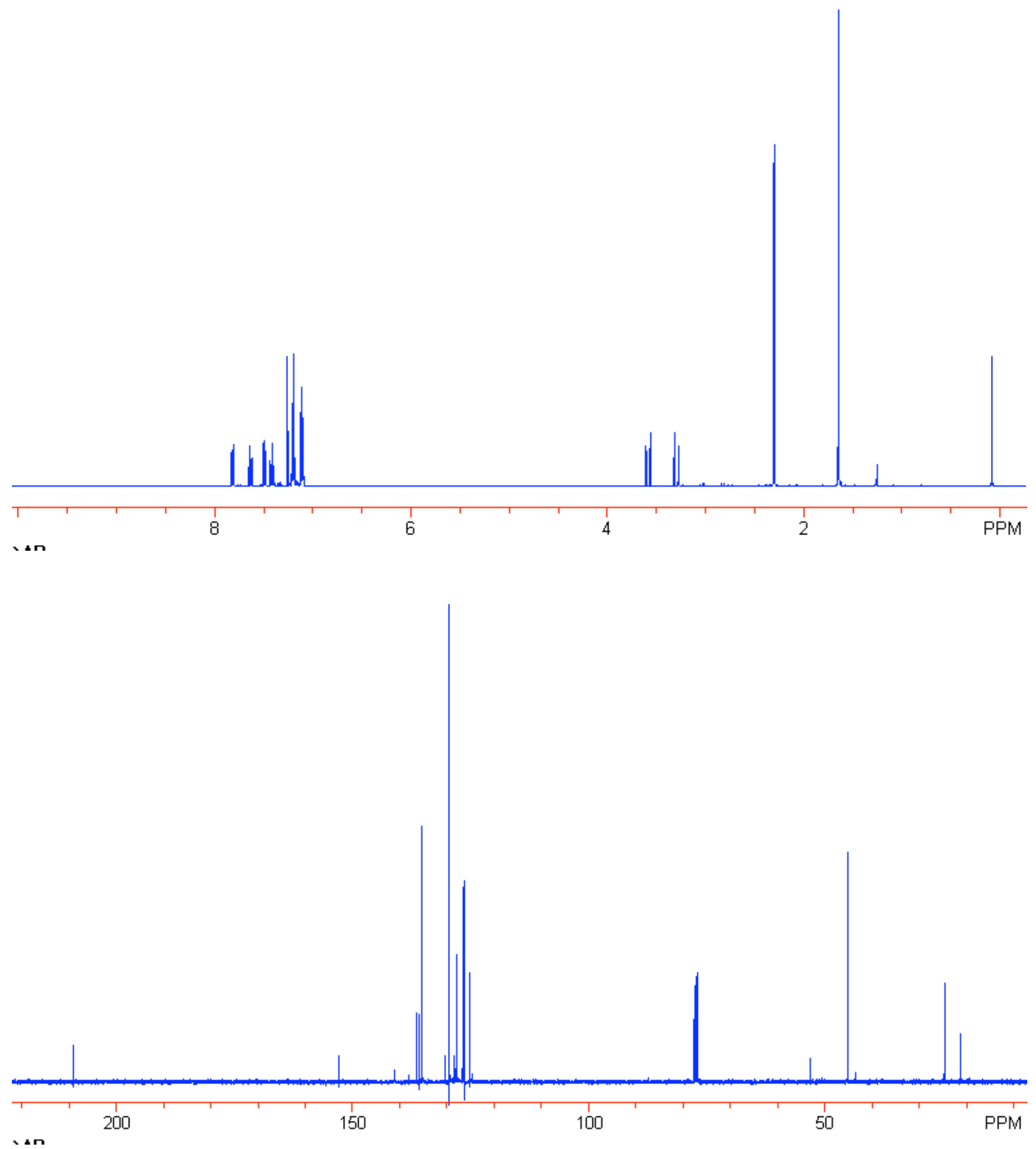

2-Methyl-2-(3,5-di-t-butylphenyl)-1-indanone (Table 2, Entry 8). Method A was followed. The reaction mixture was stirred for $40 \mathrm{~h}$ and was purified by preparative TLC (hexane/ether $=$ 97:3) to provide the title compound as a white solid in $84 \%$ yield. . The ee was determined to be $89 \%$ by chiral HPLC analysis (Chiralcel AD-H column; solvent, hexane/isopropanol (98.6/1.4); 
flow rate, $0.50 \mathrm{~mL} / \mathrm{min}$; UV lamp, $254 \mathrm{~nm}) .[\alpha]^{26}{ }_{\mathrm{D}}=-19.3\left(\mathrm{c}=0.15, \mathrm{CHCl}_{3}\right) .{ }^{1} \mathrm{H} \mathrm{NMR}\left(\mathrm{CDCl}_{3}\right.$, $500 \mathrm{MHz}): \delta 1.30(18 \mathrm{H}, \mathrm{s}), 1.68(3 \mathrm{H}, \mathrm{s}), 3.32(1 \mathrm{H}, \mathrm{d}, \mathrm{J}=17.1 \mathrm{~Hz}), 3.63(1 \mathrm{H}, \mathrm{d}, \mathrm{J}=17.4 \mathrm{~Hz})$, $7.18(2 \mathrm{H}, \mathrm{d}, \mathrm{J}=1.8 \mathrm{~Hz}), 7.31(1 \mathrm{H}, \mathrm{t}, \mathrm{J}=1.7 \mathrm{~Hz}), 7.43(1 \mathrm{H}, \mathrm{t}, \mathrm{J}=7.5 \mathrm{~Hz}), 7.50(1 \mathrm{H}, \mathrm{d}, \mathrm{J}=7.7$ $\mathrm{Hz}), 7.65(1 \mathrm{H}, \mathrm{td}, \mathrm{J}=7.5,1.1 \mathrm{~Hz}), 7.84(1 \mathrm{H}, \mathrm{d}, \mathrm{J}=7.7 \mathrm{~Hz}) ;{ }^{13} \mathrm{C} \mathrm{NMR}\left(\mathrm{CDCl}_{3}, 125 \mathrm{MHz}\right): \delta 25.2$, 31.7, 35.2, 45.3, 53.8, 120.6, 121.0, 125.1, 126.5, 127.9, 135.2, 136.0, 143.0, 150.8, 152.8, 209.2. Anal. Calcd. For $\mathrm{C}_{24} \mathrm{H}_{30} \mathrm{O}$ : C, 86.18; H, 9.04. Found: C, 86.103; H, 9.27.

2-Methyl-2-(3,4-di-methoxylphenyl)-1-indanone (Table 2, Entry 9). Method A was followed. The reaction mixture was stirred for $50 \mathrm{~h}$ and was purified by preparative TLC (hexane/ether $=80: 20$ ) to provide the title compound in $78 \%$ yield. The ee was determined to be 82\% by chiral HPLC analysis (Chiralcel AD-H column; solvent, hexane/isopropanol (90/10); flow rate, $0.50 \mathrm{~mL} / \mathrm{min}$; UV lamp, $254 \mathrm{~nm}) .[\alpha]^{26}{ }_{\mathrm{D}}=-62.2\left(\mathrm{c}=0.50, \mathrm{CHCl}_{3}\right) .{ }^{1} \mathrm{H} \mathrm{NMR}\left(\mathrm{CDCl}_{3}\right.$, $400 \mathrm{MHz}): \delta 1.63(3 \mathrm{H}, \mathrm{s}), 3.28(1 \mathrm{H}, \mathrm{d}, \mathrm{J}=17.3 \mathrm{~Hz}), 3.57(1 \mathrm{H}, \mathrm{d}, \mathrm{J}=17.4 \mathrm{~Hz}), 3.820(3 \mathrm{H}, \mathrm{s})$, $3.823(3 \mathrm{H}, \mathrm{s}), 6.77(1 \mathrm{H}, \mathrm{d}, \mathrm{J}=8.1 \mathrm{~Hz}), 6.83(1 \mathrm{H}, \mathrm{d}, \mathrm{J}=2.2 \mathrm{~Hz}), 6.84-6.86(1 \mathrm{H}, \mathrm{m}), 7.40(1 \mathrm{H}, \mathrm{t}, \mathrm{J}$ $=7.4 \mathrm{~Hz}), 7.48(1 \mathrm{H}, \mathrm{d}, \mathrm{J}=7.5 \mathrm{~Hz}), 7.63(1 \mathrm{H}, \mathrm{td}, \mathrm{J}=7.5,1.2 \mathrm{~Hz}), 7.80(1 \mathrm{H}, \mathrm{d}, \mathrm{J}=7.7 \mathrm{~Hz}) .{ }^{13} \mathrm{C}$ NMR $\left(\mathrm{CDCl}_{3}, 100 \mathrm{MHz}\right): \delta 25.1,45.0,53.0,56.1(2$ overlapping resonances), 110.1, 111.2, 118.5, 125.1, 126.6, 128.0, 135.4, 135.7, 136.5, 148.0, 149.0, 152.7, 209.0. Anal. Calcd. For $\mathrm{C}_{18} \mathrm{H}_{18} \mathrm{O}_{3}: \mathrm{C}, 76.57 ; \mathrm{H}, 6.43$. Found: C, 76.33; H, 6.45.

6-Benzylidene-2-methyl-2-phenyl-cyclohexanone (Table 2, Entry 10). Method A was followed. The reaction mixture was stirred for $60 \mathrm{~h}$ and was purified by preparative TLC (hexane/ether $=97: 3$ ) to provide the title compound in $80 \%$ yield. . The ee was determined to be 78\% by chiral HPLC analysis (Chiralcel AD-H column; solvent, hexane/isopropanol (98/2); flow rate, $0.50 \mathrm{~mL} / \mathrm{min}$; UV lamp, $254 \mathrm{~nm}) .[\alpha]^{26}=164\left(\mathrm{c}=0.40, \mathrm{CHCl}_{3}\right) .{ }^{1} \mathrm{H}$ NMR $\left(\mathrm{CDCl}_{3}, 400\right.$ MHz): $\delta 1.50(3 \mathrm{H}, \mathrm{s}), 1.62-1.77(2 \mathrm{H}, \mathrm{m}), 1.97-2.05(1 \mathrm{H}, \mathrm{m}), 2.48-2.55(1 \mathrm{H}, \mathrm{m}), 2.70-2.79(1 \mathrm{H}$, 
$\mathrm{m}), 2.83-2.89(1 \mathrm{H}, \mathrm{m}), 7.18-7.44(10 \mathrm{H}, \mathrm{m}), 7.49-7.52(1 \mathrm{H}, \mathrm{m}) .{ }^{13} \mathrm{C} \mathrm{NMR}\left(\mathrm{CDCl}_{3}, 100 \mathrm{MHz}\right)$ : $\delta 20.0,28.2,29.1,36.9,53.0,126.6,126.7,128.5,128.6,128.9,130.4,136.1,136.5,137.7$, 144.2, 205.7. Anal. Calcd. For $\mathrm{C}_{20} \mathrm{H}_{20} \mathrm{O}$ : C, 86.92; H, 7.2. Found: C, 86.793; H, 7.03.

6-Benzylidene-2-methyl-2-(3,4-di-methoxyphenyl)-cyclohexanone (Table 2, Entry 11). Method A was followed. The reaction mixture was stirred for $72 \mathrm{~h}$ and was purified by preparative TLC (hexane/ether $=80: 20$ ) to provide the title compound in 70\% yield. . The ee was determined to be $77 \%$ by chiral HPLC analysis (Chiralcel AD-H column; solvent, hexane/isopropanol (90/10); flow rate, $0.50 \mathrm{~mL} / \mathrm{min}$; UV lamp, $254 \mathrm{~nm}) .[\alpha]^{26}=84.7(\mathrm{c}=0.43$, $\left.\mathrm{CHCl}_{3}\right) .{ }^{1} \mathrm{H} \mathrm{NMR}\left(\mathrm{CDCl}_{3}, 400 \mathrm{MHz}\right): \delta 1.46(3 \mathrm{H}, \mathrm{s}), 1.67-1.77(2 \mathrm{H}, \mathrm{m}), 1.94-2.05(1 \mathrm{H}, \mathrm{m}), 2.44-$ $2.50(1 \mathrm{H}, \mathrm{m}), 2.68-2.77(1 \mathrm{H}, \mathrm{m}), 2.81-2.89(1 \mathrm{H}, \mathrm{m}), 3.86(3 \mathrm{H}, \mathrm{s}), 3.87(3 \mathrm{H}, \mathrm{s}), 6.76-6.83(3 \mathrm{H}$, $\mathrm{m})$, 7.27-7.39 (5H, m), 7.46-7.49 (1H, m). ${ }^{13} \mathrm{C} \mathrm{NMR}\left(\mathrm{CDCl}_{3}, 100 \mathrm{MHz}\right): \delta 20.0,28.3,29.1,36.8$, 50.6, 56.0, 56.1, 110.0, 111.3, 118.7, 128.5, 128.6, 130.4, 136.1, 136.30, 136.34, 138.0, 147.7, 149.1, 205.8. 

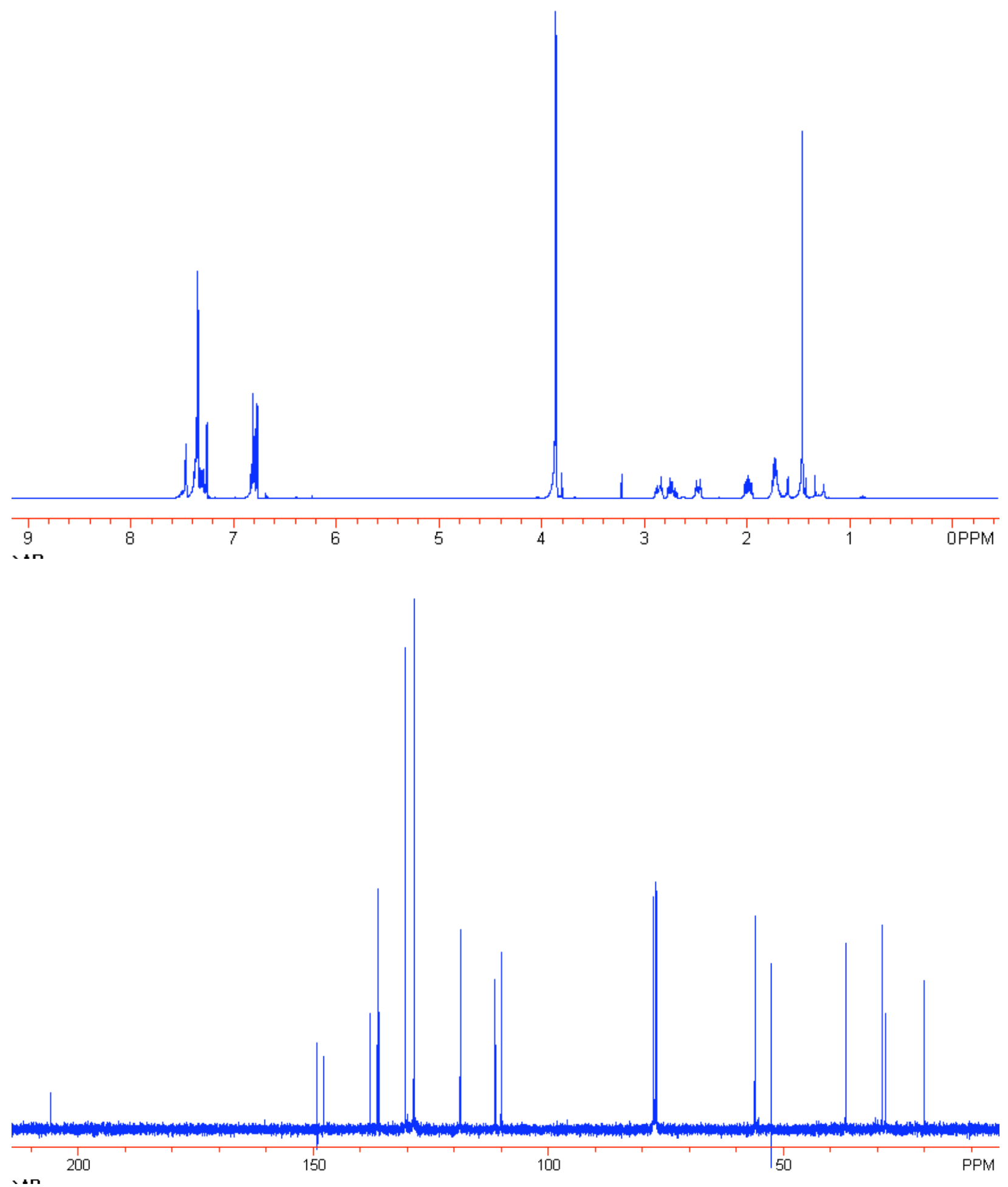

5-Benzylidene-2-methyl-2-phenyl-cyclopentanone (Table 2, Entry 12). Method A was followed. The reaction mixture was stirred for $60 \mathrm{~h}$ and was purified by preparative TLC (hexane/ether $=97: 3$ ) to provide the title compound in $70 \%$ yield. . The ee was determined to be 
95\% by chiral HPLC analysis (Chiralcel AD-H column; solvent, hexane/isopropanol (98/2); flow rate, $0.50 \mathrm{~mL} / \mathrm{min}$; UV lamp, $254 \mathrm{~nm}) .[\alpha]^{26}=-28.3\left(\mathrm{c}=0.12, \mathrm{CHCl}_{3}\right) .{ }^{1} \mathrm{H} \mathrm{NMR}\left(\mathrm{CDCl}_{3}, 400\right.$ MHz): $\delta 1.53(3 \mathrm{H}, \mathrm{s}), 2.00-2.08(1 \mathrm{H}, \mathrm{m}), 2.63-2.70(1 \mathrm{H}, \mathrm{m}), 2.79-2.90(1 \mathrm{H}, \mathrm{m}), 2.93-3.02(1 \mathrm{H}$, m), 7.21-7.62 (11H, m). ${ }^{13} \mathrm{C} \mathrm{NMR}\left(\mathrm{CDCl}_{3}, 100 \mathrm{MHz}\right): \delta 25.4,26.5,35.8,53.3,126.7,127.0$, 128.86, 128.94, 129.7, 130.9, 134.1, 135.7, 135.9, 142.8, 209.0. 

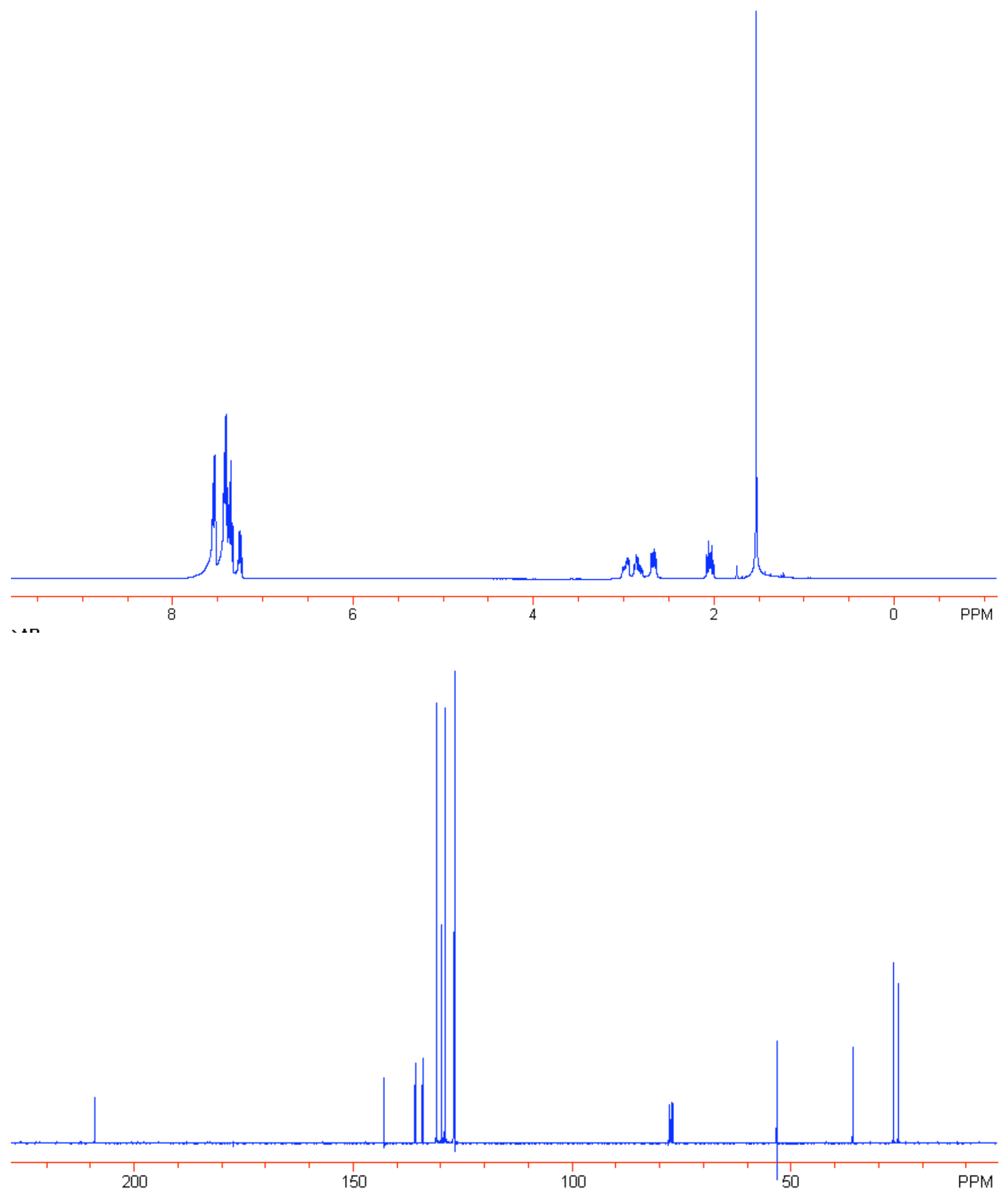

2-Methyl-2-(4-trifluoromethylphenyl)-1-indanone (Table 3, Entry 2). Method B was followed. The reaction mixture was stirred at $80{ }^{\circ} \mathrm{C}$ for $60 \mathrm{~h}$ and was purified by chromatography on silica gel, eluting with hexane/ether $(97 / 3)$ to provide the product in $69 \%$ 
yield. The ee was determined to be $96 \%$ by chiral HPLC analysis (Chiralcel OJ column; solvent, hexane/isopropanol (98/2); flow rate, $0.50 \mathrm{~mL} / \mathrm{min}$; UV lamp, $254 \mathrm{~nm}) .[\alpha]^{26}=-33.4(\mathrm{c}=0.31$, $\left.\mathrm{CHCl}_{3}\right) .{ }^{1} \mathrm{H} \mathrm{NMR}\left(\mathrm{CDCl}_{3}, 400 \mathrm{MHz}\right): \delta 1.68(3 \mathrm{H}, \mathrm{s}), 3.35(1 \mathrm{H}, \mathrm{d}, \mathrm{J}=17.6 \mathrm{~Hz}), 3.58(1 \mathrm{H}, \mathrm{d}, \mathrm{J}=$ 17.4 Hz), 7.42-7.46 (3H, m), $7.51(1 \mathrm{H}, \mathrm{d}, \mathrm{J}=7.5 \mathrm{~Hz}), 7.55(2 \mathrm{H}, \mathrm{d}, \mathrm{J}=7.1 \mathrm{~Hz}), 7.67(1 \mathrm{H}, \mathrm{td}, \mathrm{J}=$ 7.5, $1.2 \mathrm{~Hz}), 7.83(1 \mathrm{H}, \mathrm{d}, \mathrm{J}=7.4 \mathrm{~Hz}) .{ }^{13} \mathrm{C} \mathrm{NMR}\left(\mathrm{CDCl}_{3}, 100 \mathrm{MHz}\right): \delta 24.8,44.8,53.3,124.4(\mathrm{q}$, $\mathrm{J}=270.3 \mathrm{~Hz}), 125.2,125.7(\mathrm{q}, \mathrm{J}=3.8 \mathrm{~Hz}), 126.7,126.9,128.2,129.1(\mathrm{q}, \mathrm{J}=32.4 \mathrm{~Hz}), 135.4$ 135.7, 148.1, 152.5, 208.1 Anal. Calcd. For $\mathrm{C}_{17} \mathrm{H}_{13} \mathrm{~F}_{3} \mathrm{O}: \mathrm{C}, 70.34 ; \mathrm{H}, 4.51$. Found: $\mathrm{C}, 69.92 ; \mathrm{H}$, 4.56.

2-Methyl-2-(3-trifluoromethylphenyl)-1-indanone (Table 3, Entry 3). Method B was followed. The reaction mixture was stirred at $80{ }^{\circ} \mathrm{C}$ for $70 \mathrm{~h}$ and was purified by chromatography on silica gel, eluting with hexane/ether (97/3) to provide the product in $70 \%$ yield. The ee was determined to be $86 \%$ by chiral HPLC analysis (Chiralcel OJ-H column; solvent, hexane/isopropanol (97/3); flow rate, $0.50 \mathrm{~mL} / \mathrm{min}$; UV lamp, $254 \mathrm{~nm}) .[\alpha]^{26}=-30.2(\mathrm{c}$ $\left.=0.45, \mathrm{CHCl}_{3}\right) .{ }^{1} \mathrm{H} \mathrm{NMR}\left(\mathrm{CDCl}_{3}, 400 \mathrm{MHz}\right): \delta 1.67(3 \mathrm{H}, \mathrm{s}), 3.35(1 \mathrm{H}, \mathrm{d}, \mathrm{J}=17.3 \mathrm{~Hz}), 3.57(1 \mathrm{H}$, $\mathrm{d}, \mathrm{J}=17.3 \mathrm{~Hz}), 7.38-7.52(5 \mathrm{H}, \mathrm{m}), 7.61(1 \mathrm{H}, \mathrm{brs}), 7.66(1 \mathrm{H}, \mathrm{td}, \mathrm{J}=7.5,1.2 \mathrm{~Hz}), 7.83(1 \mathrm{H}, \mathrm{d}, \mathrm{J}=$ $7.8 \mathrm{~Hz}) .{ }^{13} \mathrm{C} \mathrm{NMR}\left(\mathrm{CDCl}_{3}, 100 \mathrm{MHz}\right): \delta 25.0,44.8,53.2,123.0(\mathrm{q}, \mathrm{J}=270.8 \mathrm{~Hz}), 123.2(\mathrm{q}, \mathrm{J}=$ $3.8 \mathrm{~Hz}), 123.8(\mathrm{q}, \mathrm{J}=3.8 \mathrm{~Hz}), 125.2,126.7,128.2,129.3,130.9,131.2(\mathrm{q}, \mathrm{J}=31.9 \mathrm{~Hz}), 135.4$, 135.7, 145.1, 152.4, 208.1 Anal. Calcd. For $\mathrm{C}_{17} \mathrm{H}_{13} \mathrm{~F}_{3} \mathrm{O}$ : C, 70.34; H, 4.51. Found: C, 69.99; H, 4.46 .

2-Methyl-2-(4-cyanophenyl)-1-tetralone (Table 3, Entry 4). Method C was followed. The reaction mixture was stirred at $100{ }^{\circ} \mathrm{C}$ for $80 \mathrm{~h}$ and was purified by chromatography on silica gel, eluting with hexane/ether (80/20) to provide the product in $55 \%$ yield. The ee was determined to be $97 \%$ by chiral HPLC analysis (Chiralcel OB-H column; solvent, hexane/isopropanol (80/20); 
flow rate, $1.0 \mathrm{~mL} / \mathrm{min}$; UV lamp, $254 \mathrm{~nm}) .[\alpha]^{26}=191\left(\mathrm{c}=0.44, \mathrm{CHCl}_{3}\right) .{ }^{1} \mathrm{H} \mathrm{NMR}\left(\mathrm{CDCl}_{3}, 400\right.$ MHz): $\delta 1.53(3 \mathrm{H}, \mathrm{s}), 2.25-2.32(1 \mathrm{H}, \mathrm{m}), 2.60(1 \mathrm{H}, \mathrm{dt}, \mathrm{J}=14.2,4.5 \mathrm{~Hz}), 2.73-2.80(1 \mathrm{H}, \mathrm{m}), 2.89$ $(1 \mathrm{H}, \mathrm{dt}, \mathrm{J}=17.1,4.4 \mathrm{~Hz}), 7.14(1 \mathrm{H}, \mathrm{d}, \mathrm{J}=7.8 \mathrm{~Hz}), 7.32-7.35(3 \mathrm{H}, \mathrm{m}), 7.45(1 \mathrm{H}, \mathrm{t}, \mathrm{J}=7.4 \mathrm{~Hz})$ 7.55-7.58 (2H, m), $8.14(1 \mathrm{H}, \mathrm{d}, \mathrm{J}=7.8 \mathrm{~Hz}) .{ }^{13} \mathrm{C} \mathrm{NMR}\left(\mathrm{CDCl}_{3}, 125 \mathrm{MHz}\right): \delta$ 26.1, 26.5, 36.2, 50.9, 110.9, 118.9, 127.1, 127.6, 128.4, 129.0, 132.4, 132.6, 133.9, 143.4, 148.3, 200.4.

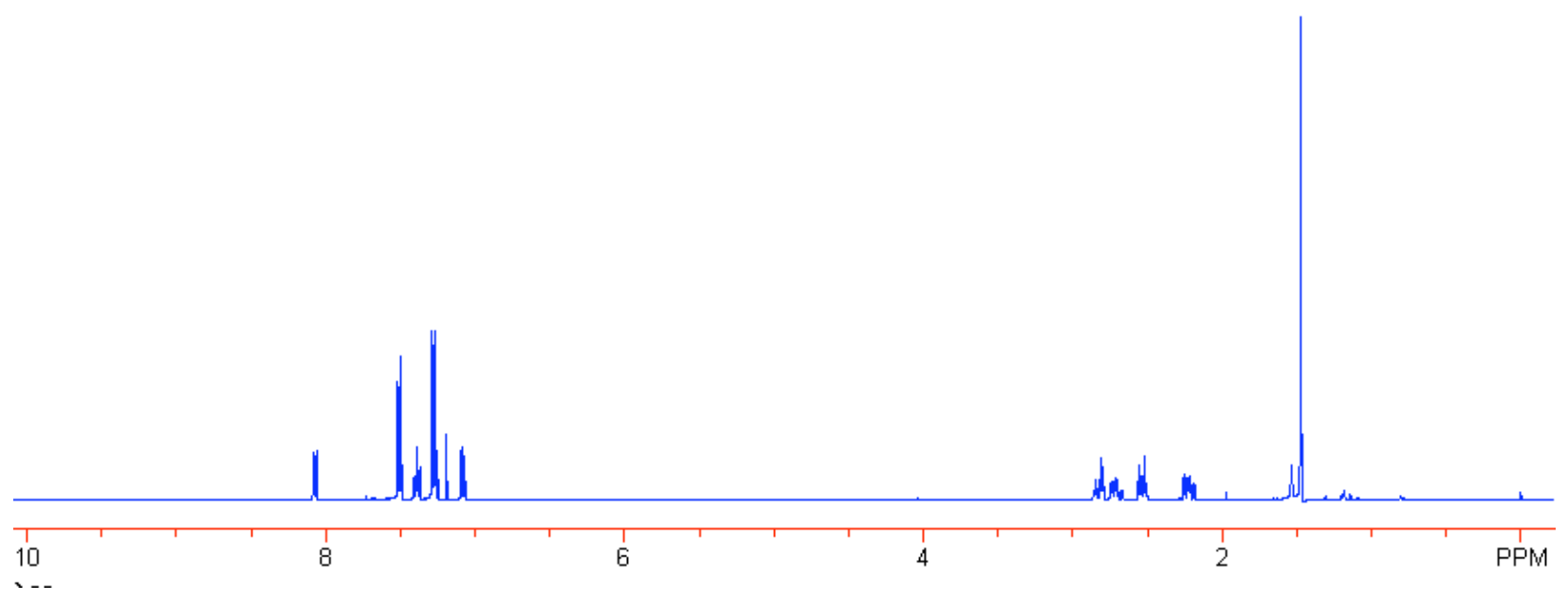




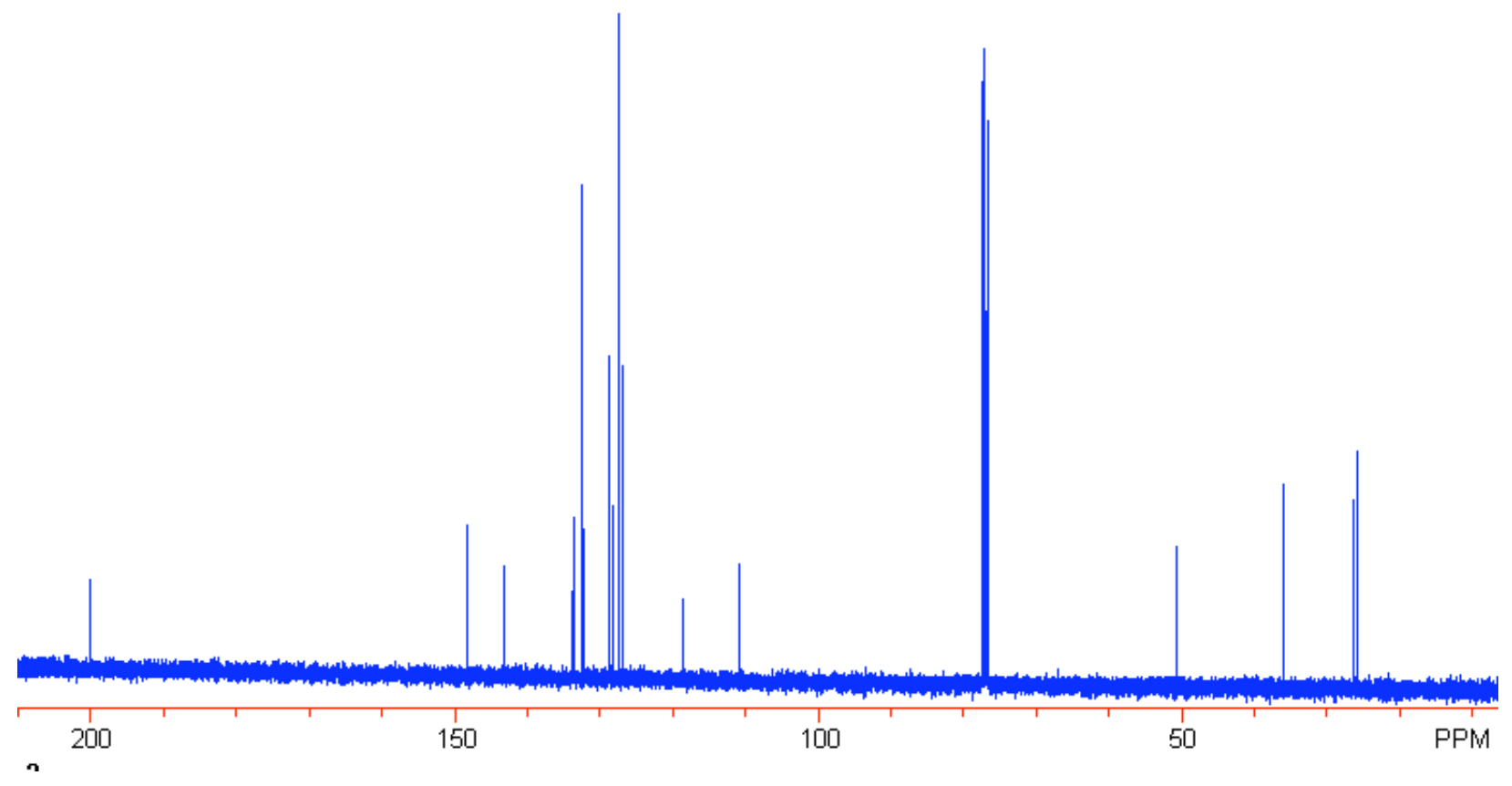

2-Methyl-2-(4-trifluoromethylphenyl)-1-tetralone (Table 3, Entry 5). Method C was followed. The reaction mixture was stirred at $100{ }^{\circ} \mathrm{C}$ for $80 \mathrm{~h}$ and was purified by chromatography on silica gel, eluting with hexane/ether (97/3) or bezene/ $\mathrm{CH}_{2} \mathrm{Cl}_{2}$ (4/1) to provide the product in $40 \%$ yield. The ee was determined to be $98 \%$ by chiral HPLC analysis (Chiralcel OJ-H column; solvent, hexane/isopropanol (98/2); flow rate, $0.5 \mathrm{~mL} / \mathrm{min}$; UV lamp, $254 \mathrm{~nm}$ ). $[\alpha]^{26}{ }_{\mathrm{D}}=162\left(\mathrm{c}=0.35, \mathrm{CHCl}_{3}\right) .{ }^{1} \mathrm{H} \mathrm{NMR}\left(\mathrm{CDCl}_{3}, 400 \mathrm{MHz}\right): \delta 1.56(3 \mathrm{H}, \mathrm{s}), 2.25-2.34(1 \mathrm{H}, \mathrm{m})$, $2.63(1 \mathrm{H}, \mathrm{dt}, \mathrm{J}=14.0,4.3 \mathrm{~Hz}), 2.76-2.92(2 \mathrm{H}, \mathrm{m}), 7.14(1 \mathrm{H}, \mathrm{d}, \mathrm{J}=7.5 \mathrm{~Hz}), 7.31-7.37(3 \mathrm{H}, \mathrm{m})$, $7.44(1 \mathrm{H}, \mathrm{td}, \mathrm{J}=7.4,1.4 \mathrm{~Hz}), 7.53(2 \mathrm{H}, \mathrm{d}, \mathrm{J}=8.0 \mathrm{~Hz}), 8.16(1 \mathrm{H}, \mathrm{dd}, \mathrm{J}=7.8,1.0 \mathrm{~Hz}) .{ }^{13} \mathrm{C} \mathrm{NMR}$ $\left(\mathrm{CDCl}_{3}, 100 \mathrm{MHz}\right): \delta 26.2,26.8,36.3,50.8,124.3(\mathrm{q}, \mathrm{J}=270.6 \mathrm{~Hz}), 125.7(\mathrm{q}, \mathrm{J}=3.8 \mathrm{~Hz}), 127.0$, 127.1, 128.3, 129.0, 129.2 (q, J = 32.4 Hz), 132.5, 133.7, 143.5, 146.7, 200.8. 


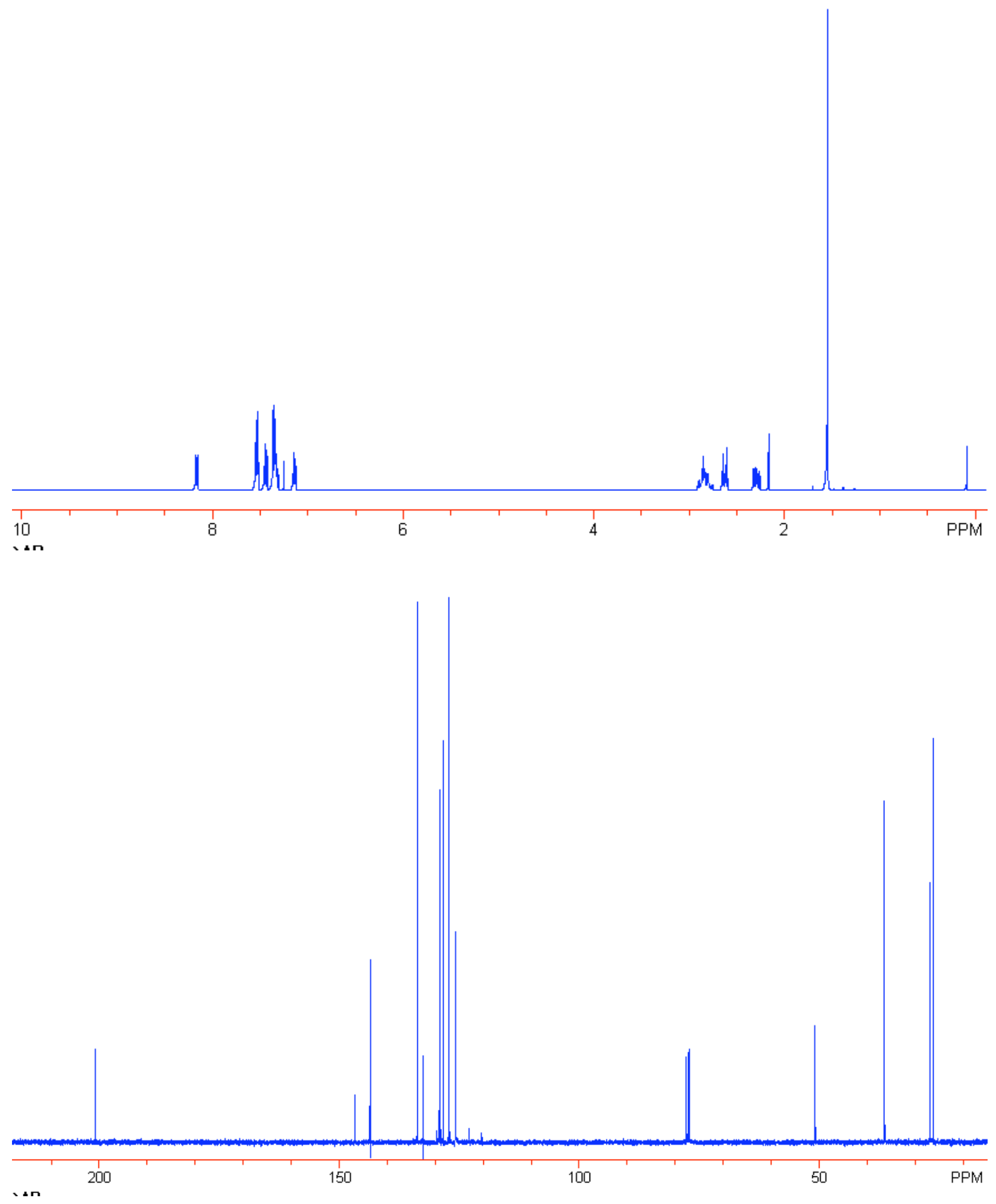




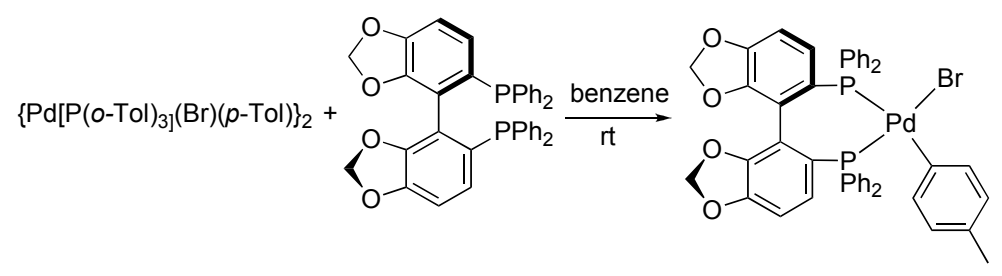

Preparation of Pd(segphos)(p-tol)(Br). To a solution of Segphos (100mg, 0.164mmol) in benzene $(7.40 \mathrm{~mL})$ was added $\left\{\mathrm{Pd}\left[\mathrm{P}(o-\mathrm{Tol})_{3}\right](\mathrm{Br})(p-\mathrm{Tol})\right\}_{2}{ }^{1}(95.4 \mathrm{mg}, 0.0820 \mathrm{mmol})$. The mixture was then stirred at room temperature for $1 \mathrm{~h}$. The resulting solution was filtered through Celite, and the volatile materials were evaporated under vacuum to provide a yellow solid. The crude product was washed with ether and further recrystallized with by layering a $\mathrm{CH}_{2} \mathrm{Cl}_{2}$ solution of the complex with hexane and cooling at $-30{ }^{\circ} \mathrm{C}$ to provide a yellow solid $(72.8 \mathrm{mg})$ in $50 \%$ yield. ${ }^{1} \mathrm{H}$ NMR $\left(\mathrm{C}_{6} \mathrm{D}_{6}, 400 \mathrm{MHz}\right): \delta 2.02(3 \mathrm{H}, \mathrm{s}), 4.91(1 \mathrm{H}, \mathrm{s}), 5.06(1 \mathrm{H}, \mathrm{s}), 5.22(1 \mathrm{H}, \mathrm{s})$, $5.23(1 \mathrm{H}, \mathrm{s}), 5.92(1 \mathrm{H}, \mathrm{d}, \mathrm{J}=8.0 \mathrm{~Hz}), 6.13(1 \mathrm{H}, \mathrm{d}, \mathrm{J}=7.2 \mathrm{~Hz}), 6.37(1 \mathrm{H}, \mathrm{t}, \mathrm{J}=8.6 \mathrm{~Hz}), 6.67-7.15$ $(15 \mathrm{H}, \mathrm{m}), 7.27-7.33(2 \mathrm{H}, \mathrm{m}), 7.74-7.84(2 \mathrm{H}, \mathrm{m}), 7.86-8.05(4 \mathrm{H}, \mathrm{m}), 8.19-8.25(2 \mathrm{H}, \mathrm{m}) .{ }^{31} \mathrm{P}\left\{{ }^{1} \mathrm{H}\right\}$ NMR (benzene) $\delta 9.0(\mathrm{~d}, \mathrm{~J}=42.6 \mathrm{~Hz}), 24.6(\mathrm{~d}, \mathrm{~J}=42.7 \mathrm{~Hz})$. Anal. Calcd. For $\mathrm{C}_{45} \mathrm{H}_{35} \mathrm{BrO}_{4} \mathrm{P}_{2} \mathrm{Pd}$ : C, 60.86; H, 3.97. Found: C, 61.15; H, 4.29 .

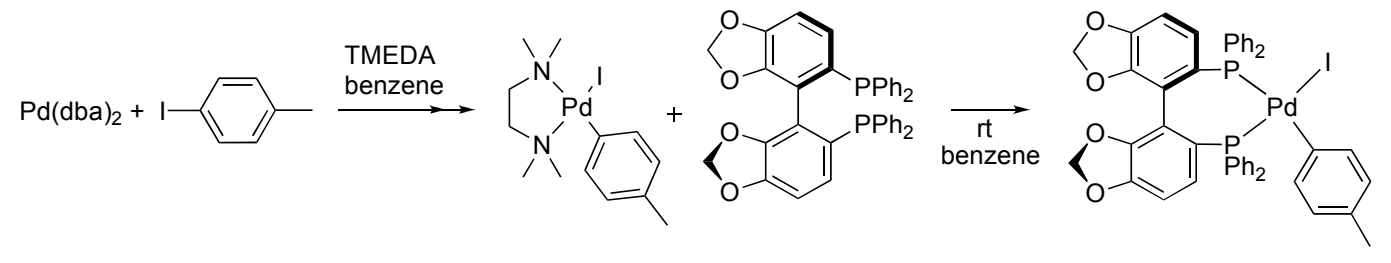

Preparation of Pd(segphos)(p-tol)(I). To a purple solution of $\mathrm{Pd}(\mathrm{dba})_{2}(0.500 \mathrm{~g}, 0.880$ mmol) in $25 \mathrm{ml}$ of benzene was added TMEDA $(0.160 \mathrm{~mL}, 1.13 \mathrm{mmol})$ and $p$-iodotoluene (273 $\mathrm{mg}, 1.25 \mathrm{mmol}$ ). The mixture was slowly heated to $50{ }^{\circ} \mathrm{C}$, during which time, the color changed to green. After filtration of the solution through Celite, the volatile materials were evaporated under reduced pressure, and the residue was washed with ether. The crude product was recrystallized by layering a $\mathrm{CH}_{2} \mathrm{Cl}_{2}$ solution of the complex with ether and cooling at $-30{ }^{\circ} \mathrm{C}$ to 
provide $\mathrm{PdI}\left(p\right.$-tol)(TMEDA) in $70 \%$ yield as a yellow solid. ${ }^{1} \mathrm{H}$ NMR $\left(\mathrm{CDCl}_{3}, 500 \mathrm{MHz}\right): \delta 2.21$ $(3 \mathrm{H}, \mathrm{s}), 2.30(6 \mathrm{H}, \mathrm{s}), 2.53(2 \mathrm{H}, \mathrm{t}, \mathrm{J}=5.3 \mathrm{~Hz}), 2.64(6 \mathrm{H}, \mathrm{s}), 2.69(2 \mathrm{H}, \mathrm{t}, \mathrm{J}=5.3 \mathrm{~Hz}), 6.75(2 \mathrm{H}, \mathrm{d}, \mathrm{J}$ $=8.0 \mathrm{~Hz}), 7.09(2 \mathrm{H}, \mathrm{d}, \mathrm{J}=7.5 \mathrm{~Hz}) .{ }^{13} \mathrm{C} \mathrm{NMR}\left(\mathrm{CDCl}_{3}, 125 \mathrm{MHz}\right): \delta 20.9,50.0,50.1,58.5,62.3$, 127.8, 131.8, 136.3, 140.0. Anal. Calcd. For $\mathrm{C}_{13} \mathrm{H}_{23} \mathrm{~N}_{2} \mathrm{IPd}$ : C, 35.43; H, 5.26; N, 6.36. Found: $\mathrm{C}$, 35.21, H, 5.17, N, 6.27.

To a solution of Segphos $(61.0 \mathrm{mg}, 0.100 \mathrm{mmol})$ in benzene $(10 \mathrm{~mL})$ was added $\operatorname{PdI}(p-$ tol)(TMEDA) (40.0 mg, $0.0907 \mathrm{mmol})$. The mixture was stirred at room temperature for $30 \mathrm{~min}$, after which time $20 \mathrm{~m}$ of pentane was added to afford a yellow precipitate. The resulting mixture was filtered to provide the crude product as a yellow solid. This solid was then washed with ether and dried in vacuo (33.5 mg, $40 \%$ yield). ${ }^{1} \mathrm{H}$ NMR $\left(\mathrm{C}_{6} \mathrm{D}_{6}, 400 \mathrm{MHz}\right): \delta 2.06(3 \mathrm{H}, \mathrm{s}), 4.87-$ $4.91(1 \mathrm{H}, \mathrm{m}), 5.01-5.05(1 \mathrm{H}, \mathrm{m}), 5.23(1 \mathrm{H}, \mathrm{d}, \mathrm{J}=1.4 \mathrm{~Hz}), 5.24(1 \mathrm{H}, \mathrm{d}, \mathrm{J}=1.2 \mathrm{~Hz}), 5.96(1 \mathrm{H}, \mathrm{dd}$, $\mathrm{J}=8.0,1.0 \mathrm{~Hz}), 6.19(1 \mathrm{H}, \mathrm{dd}, \mathrm{J}=8.1,1.4 \mathrm{~Hz}), 6.50(1 \mathrm{H}, \mathrm{t}, \mathrm{J}=8.5 \mathrm{~Hz}), 6.66-7.20(15 \mathrm{H}, \mathrm{m})$, 7.26-7.34 (2H, m), $7.80(2 \mathrm{H}, \mathrm{td}, \mathrm{J}=7.9,2.6 \mathrm{~Hz}), 7.87-8.00(4 \mathrm{H}, \mathrm{m}), 8.17-8.25(2 \mathrm{H}, \mathrm{m}) .{ }^{31} \mathrm{P}\left\{{ }^{1} \mathrm{H}\right\}$ NMR (benzene) $\delta 9.9(\mathrm{~d}, \mathrm{~J}=43.9 \mathrm{~Hz}), 21.3\left(\mathrm{~d}, \mathrm{~J}=45.2 \mathrm{~Hz}\right.$ ). Anal. Calcd. For $\mathrm{C}_{45} \mathrm{H}_{35} \mathrm{IO}_{4} \mathrm{P}_{2} \mathrm{Pd}: \mathrm{C}$, 57.80; H, 3.77. Found: C, 57.70; H, 4.06.

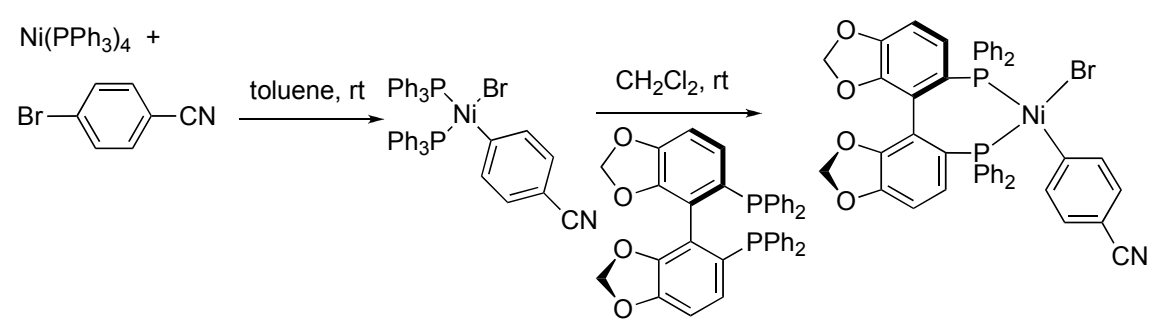

Prepapration of (segphos) Ni( $\left.\mathbf{C}_{6} \mathbf{H}_{4}-4-\mathbf{C N}\right) \mathbf{B r}$ complex. To a solution of $\mathrm{Ni}\left(\mathrm{PPh}_{3}\right)_{4}(221 \mathrm{mg}$, $0.200 \mathrm{mmol})$ in toluene $(2 \mathrm{~mL})$ was added 4-bromobenzonitrile $(182 \mathrm{mg}, 1.00 \mathrm{mmol})$ in toluene $(2 \mathrm{~mL})$. The mixture was stirred at room temperature for $2.5 \mathrm{~h}$ at which time full conversion was achieved, according to ${ }^{31} \mathrm{P}$ NMR spectroscopy. The resulting light-brown suspension was filtered, washed with ether and pentane, and dried in vacuo to provide $\left(\mathrm{PPh}_{3}\right)_{2} \mathrm{Ni}\left(\mathrm{C}_{6} \mathrm{H}_{4}-4-\mathrm{CN}\right) \mathrm{Br}$ 
as a yellow solid (115 mg, 75\% yield). ${ }^{1} \mathrm{H} \mathrm{NMR}\left(\mathrm{CD}_{2} \mathrm{Cl}_{2}, 500 \mathrm{MHz}\right): \delta 6.33(2 \mathrm{H}$, broad s), 6.90$7.05(2 \mathrm{H}, \mathrm{m}), 7.42-7.55(18 \mathrm{H}, \mathrm{m}), 7.63\left(12 \mathrm{H}\right.$, broad s). ${ }^{31} \mathrm{P}\left\{{ }^{1} \mathrm{H}\right\} \mathrm{NMR}\left(\mathrm{CD}_{2} \mathrm{Cl}_{2}\right) \delta 24.0$. Anal. Calcd. For $\mathrm{C}_{43} \mathrm{H}_{34} \mathrm{BrNNiP}_{2}$ : C, 67.49; H, 4.48; N, 1.83. Found: C, 67.54; H, 4.50; N, 1.93 .

A mixture of $\mathrm{Ni}(\mathrm{Br})\left(\mathrm{C}_{6} \mathrm{H}_{4} \mathrm{CN}\right)\left(\mathrm{PPh}_{3}\right)_{2}(62 \mathrm{mg}, 0.081 \mathrm{mmol})$ and segphos $(50 \mathrm{mg}, 0.082$ mmol) were dissolved in $\mathrm{CH}_{2} \mathrm{Cl}_{2}(2 \mathrm{~mL})$, and the resulting solution was stirred at room temperature for $25 \mathrm{~min}$. Ether $(2 \mathrm{~mL})$ was then added to the reaction mixture, followed by pentane $(5 \mathrm{~mL})$. The resulting mixture was then kept at $-35{ }^{\circ} \mathrm{C}$ for $20 \mathrm{~h}$ to afford (segphos) $\mathrm{Ni}\left(\mathrm{C}_{6} \mathrm{H}_{4}-4-\mathrm{CN}\right) \mathrm{Br}$ as dark brown crystals $\left(34 \mathrm{mg}, 49 \%\right.$ yield). ${ }^{1} \mathrm{H} \mathrm{NMR}\left(\mathrm{CDCl}_{3}, 500\right.$ $\mathrm{MHz}): \delta 5.45(1 \mathrm{H}$, broad s), $5.52(1 \mathrm{H}$, broad s), $5.79(2 \mathrm{H}, \mathrm{s}), 6.48(2 \mathrm{H}, \mathrm{s}), 6.81(2 \mathrm{H}, \mathrm{d}, \mathrm{J}=8.0$ Hz), 7.11-7.59 (18 H, m), 7.69 (2H, broad s), 7.83-8.01 (4H, m). ${ }^{31} \mathrm{P}\left\{{ }^{1} \mathrm{H}\right\} \mathrm{NMR}\left(\mathrm{CDCl}_{3}\right) \delta 14.1$ $(\mathrm{d}, \mathrm{J}=42.7 \mathrm{~Hz}), 26.1(\mathrm{~d}, \mathrm{~J}=45.9 \mathrm{~Hz})$. Anal. Calcd. For $\left.\mathrm{C}_{45} \mathrm{H}_{32} \mathrm{BrNNiO}_{4} \mathrm{P}_{2} \bullet 0.5 \mathrm{CH}_{2} \mathrm{Cl}_{2} \bullet 0.5 \mathrm{Et}_{2} \mathrm{O}\right)$ : C, 61.29; $\mathrm{H}, 4.11 ; \mathrm{N}, 1.50$. Found: $\mathrm{C}, 61.40 ; \mathrm{H}, 3.98 ; \mathrm{N}, 1.63$. The ratio of $\mathrm{CH}_{2} \mathrm{Cl}_{2}$ and $\mathrm{Et}_{2} \mathrm{O}$ to the nickel complex was determined by ${ }^{1} \mathrm{H}$ NMR spectroscopy.

Stoichiometric reactions of the arylmetal halide complexes:

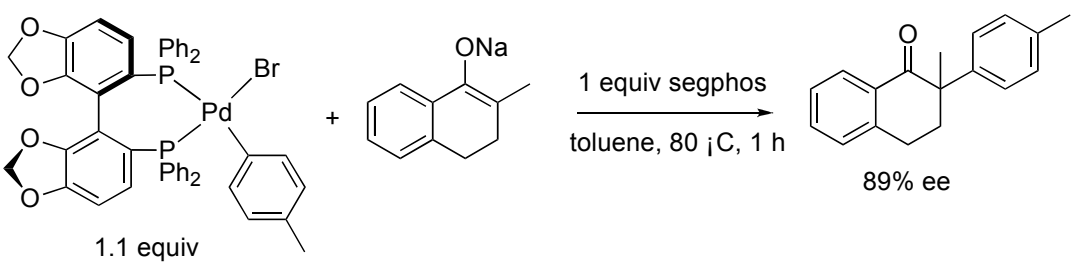

Reaction of Pd(segphos)(p-tol)(Br), and the sodium enolate of 2-methyl-1-tetralone: To a screw-capped vial containing segphos $(20.6 \mathrm{mg}, 0.0338 \mathrm{mmol}), \mathrm{Pd}(\mathrm{segphos})(p$-tol $)(\mathrm{Br})(30.0 \mathrm{mg}$, $0.0338 \mathrm{mmol})$, and the sodium enolate of 2-methyl-1-tetralone $(6.2 \mathrm{mg}, 0.0338 \mathrm{mmol})$ was added toluene $(0.675 \mathrm{~mL})$. The vial was sealed with a cap containing a PTFE septum and removed from drybox. The reaction mixture was stirred at $80{ }^{\circ} \mathrm{C}$ for $1 \mathrm{~h}$. The same workup, purification and 
Chiral HPLC conditions were then followed as for the catalytic reactions. The yield of product was $90 \%$ and ee of the product was $89 \%$.

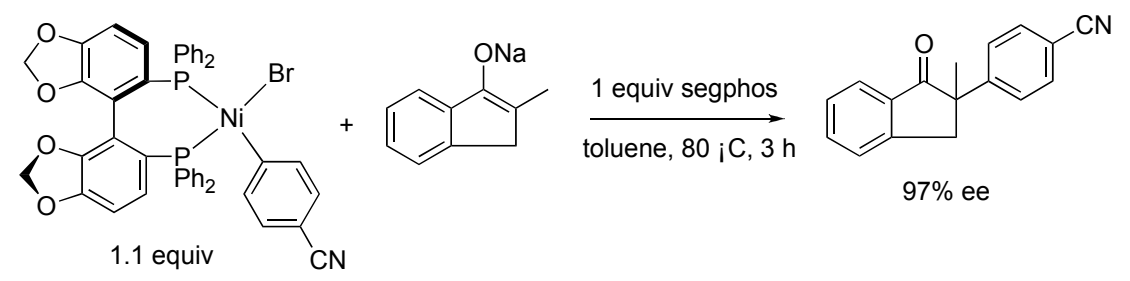

Reaction of (segphos) Ni( $\left.\mathrm{C}_{6} \mathrm{H}_{4}-4-\mathrm{CN}\right) \mathrm{Br}$ with the sodium enolate of 2-methyl-1-tetralone: To a screw-capped vial containing segphos (33.3 mg, $0.0546 \mathrm{mmol}$ ), (segphos) Ni( $\left.\mathrm{C}_{6} \mathrm{H}_{4}-4-\mathrm{CN}\right) \mathrm{Br}$ complex (46.5 mg, $0.0546 \mathrm{mmol})$ and the sodium enolate of 2-methyl-1-indanone $(9.1 \mathrm{mg}, 0.055$ mmol) was added toluene $(0.440 \mathrm{~mL})$. The vial was sealed with a cap containing a PTFE septum and removed from drybox. The reaction mixture was stirred at $80^{\circ} \mathrm{C}$ for $3 \mathrm{~h}$. The same workup, purification and Chiral HPLC conditions were followed as for the catalytic reactions. The yield of the product was $90 \%$ and ee of the product was $97 \%$.

\section{Determination of Absolute Configuration:}

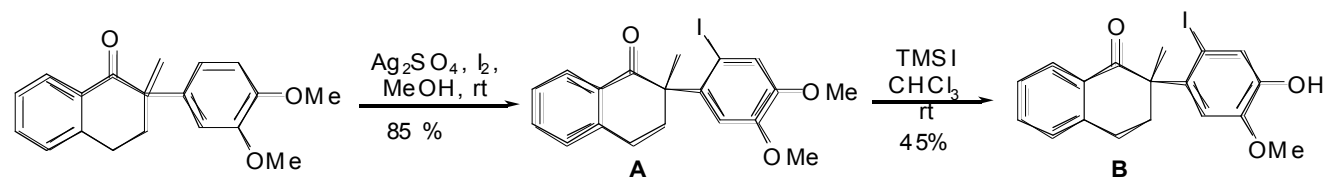

Iodination: To a solution of 2-methyl-2-(3,4-di-methoxylphenyl)-1-tetralone ${ }^{3}$ (50.0 mg, $0.168 \mathrm{mmol})$ in $\mathrm{MeOH}(5 \mathrm{~mL})$ was added $\mathrm{Ag}_{2} \mathrm{SO}_{4}(57.5 \mathrm{mg}, 0.185 \mathrm{mmol})$ and $\mathrm{I}_{2}(47.0 \mathrm{mg}, 0.185$ $\mathrm{mmol}$ ) at room temperature. The mixture was stirred for $4 \mathrm{~h}$ at room temperature. The resulting mixture was filtered through Celite to provide a clear solution. The solvent was evaporated under vacuum to provide crude product. The crude product was purified by chromatography on silica gel, eluting with hexane/ether (4/1) to provide the product $\mathbf{A}$ in $85 \%$ yield. ${ }^{1} \mathrm{H}$ NMR $\left(\mathrm{CDCl}_{3}, 400\right.$ MHz): $\delta 1.70(3 \mathrm{H}, \mathrm{s}), 1.78-1.85(1 \mathrm{H}, \mathrm{m}), 2.93-3.02(1 \mathrm{H}, \mathrm{m}), 3.14-3.30(2 \mathrm{H}, \mathrm{m}), 3.84(3 \mathrm{H}, \mathrm{s})$, 
$3.85(3 \mathrm{H}, \mathrm{s}), 6.91(1 \mathrm{H}, \mathrm{s}), 7.24(1 \mathrm{H}, \mathrm{d}, \mathrm{J}=7.6 \mathrm{~Hz}), 7.33(1 \mathrm{H}, \mathrm{s}), 7.35(1 \mathrm{H}, \mathrm{t}, \mathrm{J}=7.4 \mathrm{~Hz}), 7.49$

$(1 \mathrm{H}, \mathrm{td}, \mathrm{J}=7.3,1.5 \mathrm{~Hz}), 8.18(1 \mathrm{H}, \mathrm{dd}, \mathrm{J}=7.7,1.5 \mathrm{~Hz}) .{ }^{13} \mathrm{C} \mathrm{NMR}\left(\mathrm{CDCl}_{3}, 125 \mathrm{MHz}\right): \delta 22.8$, $26.2,35.4,52.8,56.1,56.3,86.2,112.9,124.9,127.1,128.4,129.1,133.3,133.6,139.5,141.8$, 148.1, 148.9, 200.0.

Demethylation of the Aryl Methyl Ether": To a solution of iodo compound A (50.0 mg, $0.118 \mathrm{mmol})$ in $\mathrm{CHCl}_{3}(0.5 \mathrm{~mL})$ was added TMSI $(17.7 \mu \mathrm{L})$ under an inert atmosphere. This mixture was stirred at room temperature for $48 \mathrm{~h}$, after which time the reaction mixture was diluted with $5 \mathrm{~mL}$ of methanol. To this solution was added $10 \mathrm{~mL}$ of brine, and the resulting solution was extracted with ether $(2 \times 15 \mathrm{~mL})$. The extract was washed with sodium bisulfate and brine, and dried with $\mathrm{Na}_{2} \mathrm{SO}_{4}$. The solvent was removed under vacuum. The crude product was purified by chromatography on silica gel, eluting with hexane/ether (4/1) to provide the product $\mathbf{B}$ in $45 \%$ yield. A single crystal of $\mathbf{B}$ that was suitable for X-ray diffraction was obtained by layering a $\mathrm{CH}_{2} \mathrm{Cl}_{2}$ solution of $\mathbf{B}$ with hexane and cooling at $-20{ }^{\circ} \mathrm{C} .{ }^{1} \mathrm{H} \mathrm{NMR}\left(\mathrm{CDCl}_{3}, 400\right.$ MHz): $\delta 1.65(3 \mathrm{H}, \mathrm{s}), 1.75-1.83(1 \mathrm{H}, \mathrm{m}), 2.95-3.04(1 \mathrm{H}, \mathrm{m}), 3.13-3.33(2 \mathrm{H}, \mathrm{m}), 3.85(3 \mathrm{H}, \mathrm{s})$, $5.60(1 \mathrm{H}, \mathrm{bs}), 7.05(1 \mathrm{H}, \mathrm{s}), 7.24(1 \mathrm{H}, \mathrm{d}, \mathrm{J}=7.4 \mathrm{~Hz}), 7.31(1 \mathrm{H}, \mathrm{s}), 7.34(1 \mathrm{H}, \mathrm{t}, \mathrm{J}=7.6 \mathrm{~Hz}), 7.49$ $(1 \mathrm{H}, \mathrm{td}, \mathrm{J}=7.4,1.5 \mathrm{~Hz}), 8.18(1 \mathrm{H}, \mathrm{dd}, \mathrm{J}=7.8,1.5 \mathrm{~Hz}) .{ }^{13} \mathrm{C} \mathrm{NMR}\left(\mathrm{CDCl}_{3}, 125 \mathrm{MHz}\right): \delta 22.9$, $26.2,35.5,52.7,56.4,84.8,115.9,124.2,127.0,128.5,129.1,133.3,133.6,140.6,141.7,144.9$, 145.7, 199.9. 


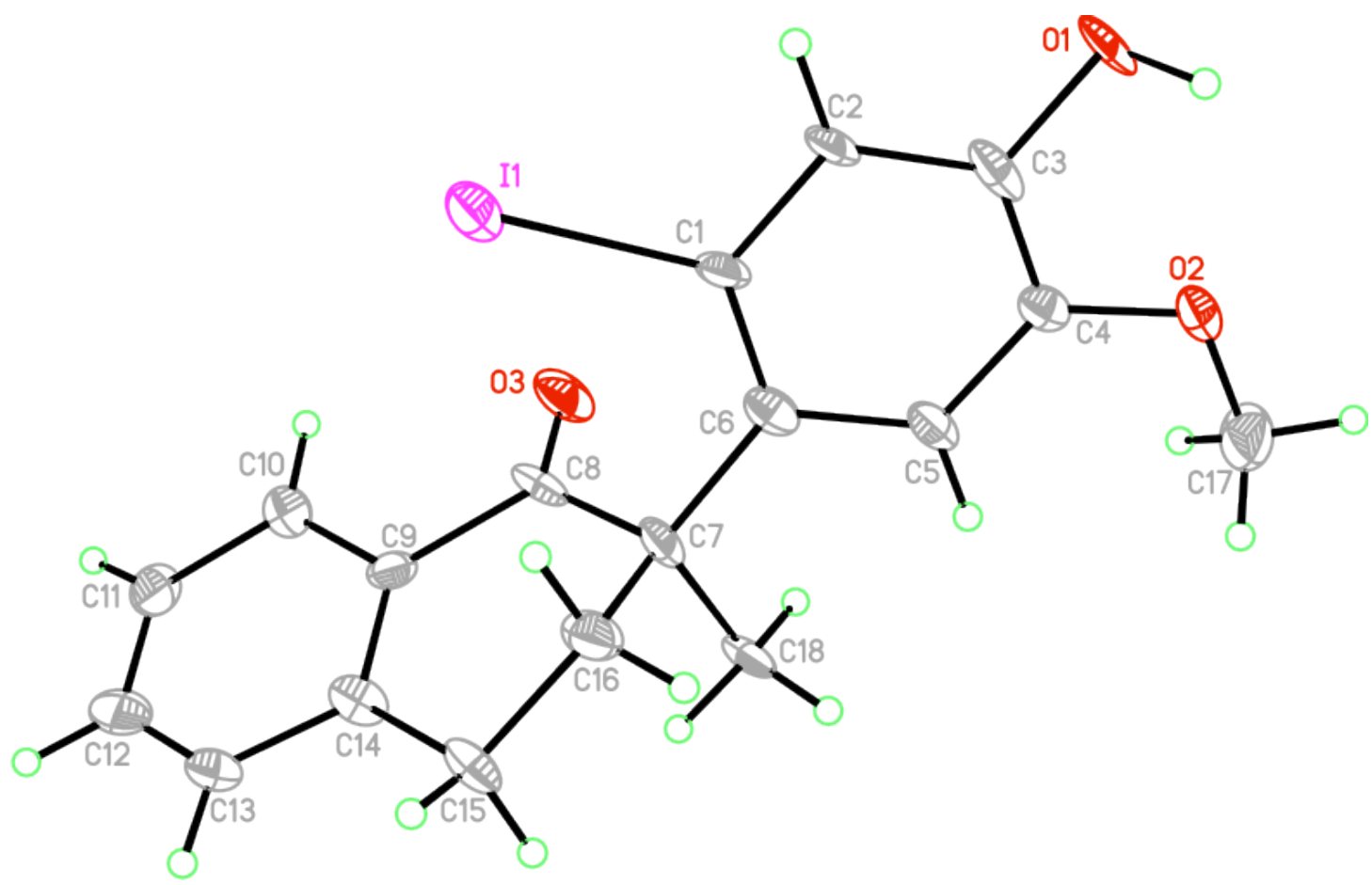

Figure S1. ORTEP digram of the des-methyl, iodo-derivative B.

\section{References:}

1. Ritter, K. Synthesis 1993, 735.

2. Paul, F.; Patt, J.; Hartwig, J. F. Organometallics 1995, 14, 3030.

3. Compound A was obtained according the condition of table 2, entry 5.

4. Vickery, E. H.; Pahler, L. F.; Eisenbraun, E. J. J. Org. Chem. 1979, 44, 4444. 